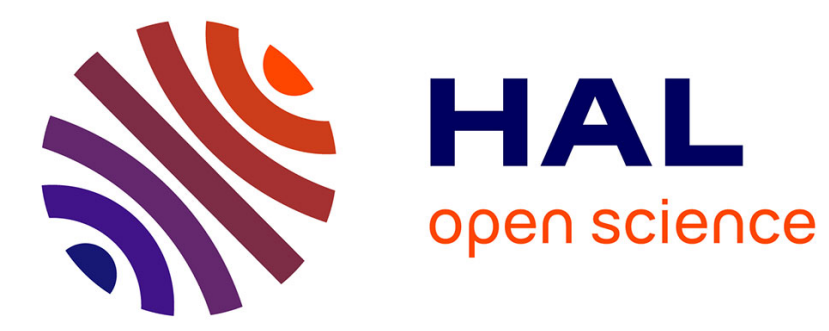

\title{
Excluded area computations for non-convex molecules Riccardo Rosso
}

\section{To cite this version:}

Riccardo Rosso. Excluded area computations for non-convex molecules. Molecular Physics, 2008, 106 (21-23), pp.2487-2506. 10.1080/00268970802575556 . hal-00513236

\section{HAL Id: hal-00513236 https://hal.science/hal-00513236}

Submitted on 1 Sep 2010

HAL is a multi-disciplinary open access archive for the deposit and dissemination of scientific research documents, whether they are published or not. The documents may come from teaching and research institutions in France or abroad, or from public or private research centers.
L'archive ouverte pluridisciplinaire HAL, est destinée au dépôt et à la diffusion de documents scientifiques de niveau recherche, publiés ou non, émanant des établissements d'enseignement et de recherche français ou étrangers, des laboratoires publics ou privés. 


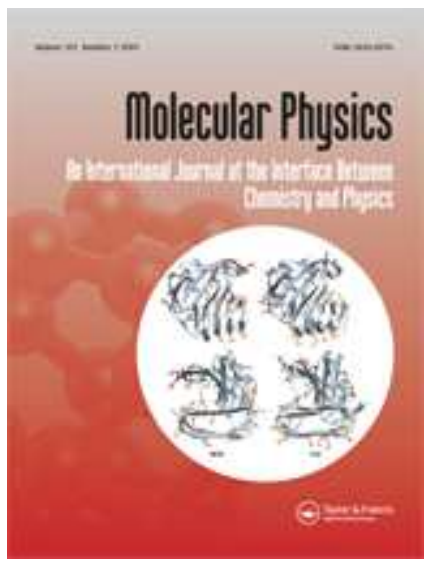

\section{Excluded area computations for non-convex molecules}

\begin{tabular}{|c|c|}
\hline Journal: & Molecular Physics \\
\hline Manuscript ID: & TMPH-2008-0242.R1 \\
\hline Manuscript Type: & Full Paper \\
\hline $\begin{array}{l}\text { Date Submitted by the } \\
\text { Author: }\end{array}$ & $21-$ Oct-2008 \\
\hline Complete List of Authors: & Rosso, Riccardo; Universita` di Pavia, Mathematics \\
\hline Keywords: & $\begin{array}{l}\text { excluded area, steric interactions, convolution curve, Minkowski } \\
\text { sum }\end{array}$ \\
\hline \multicolumn{2}{|c|}{$\begin{array}{l}\text { Note: The following files were submitted by the author for peer review, but cannot be converted } \\
\text { to PDF. You must view these files (e.g. movies) online. }\end{array}$} \\
\hline TMPH-2008-0242R1.tex & \\
\hline
\end{tabular}

\section{今 scholaroNE" \\ Manuscript Central}


where $d$ is the distance between $\mathcal{M}$ and $\mathcal{M}^{\prime}$. While tracking the excluded region and computing the excluded volume for two spherical molecules of radii $R$ and $R^{\prime}$ is easy - $\mathcal{E}$ is a sphere with radius $R+R^{\prime}$ - and so $V(\mathcal{E})=\frac{4 \pi}{3}\left(R+R^{\prime}\right)^{2}$, things become intricated when the molecular shapes are less symmetric, as in the case of liquid-crystalline compounds.

To explain the isotropic-nematic transition in colloidal suspensions forming a lyotropic liquid crystal Onsager computed explicitly the excluded volume for a pair of cylindric molecules in the now classical paper [1]. Although the predictions were later improved by Mayer-Saupe approach which completely neglects steric contributions in favour of dispersion interactions, Onsager's approach has been applied to more realistic nematogenic molecules. An early generalization was the analytic computation of $\mathcal{E}$ for a pair of ellipsoids of revolution by Isihara [2] through a format that relies on classical mathematical work on convex geometry, also used by Kihara [3] who obtained the formal expression of the excluded volume for a pair of equal convex bodies. A step forward was moved by Tjipto-Margo and Evans [4] who obtained a closed form for the excluded volume of biaxial ellipsoids which, at variance with Isihara's case, are only endowed with $D_{2 h}$, instead of $D_{\infty h}$, symmetry. Both Kihara's and Tjipto-Margo and Evans' computations have been simplified by Singh and Kumar [5], to which the reader is referred for a self-consistent account of convexbody coordinates. Another avenue has been followed by Mulder who computed explicitly the excluded volume for spheroplatelets [6] and spherocuboids [7]. In his computations the bodies in contact are viewed as the Minkowski sum of other bodies, a property that makes the powerful techniques of convex-body analysis work efficiently. In the past decade, however, the hypothesis of convex nematogenic molecules became untenable to study compounds formed, for instance, by $V$-shaped molecules. To tackle the technical problem of computing the excluded volume for a pair of non convex $V$-shaped molecules, Mulder et al. [8] resorted to interpolation. Precisely, they computed the excluded volume only on a finite set of configurations and used the results to interpolate the excluded volume when the relative orientation is arbitrary.

In [7] Mulder proved a theorem that relates the excluded region $\mathcal{E}$ to the Minkowski sum of $\mathcal{M}$ and $\ominus \mathcal{M}^{\prime}$, where $\ominus \mathcal{M}^{\prime}$ is obtained from $\mathcal{M}^{\prime}$ by a central inversion about the reference point $O^{\prime}$. This theorem is not limited to convex bodies, and it was known in a totally different community, namely that of computer-aided geometric design (CAGD, in short): see, e.g. [9]. In fact, excluded regions have been widely studied for the past three decades [10], since they are important in problems of robot motion-planning. It should be noted that the main concern it the CAGD community is not the explicit computation of the excluded volume $V(\mathcal{E})$, but a precise identification of the boundary $\partial \mathcal{E}$ of 
the excluded region. However, since the restriction to convex shapes would be too narrow for realistic applications in CAGD, several attempts have been made to extract information on $\mathcal{E}$ when the interacting-or, in this context, colliding-bodies fail to be convex. In particular, it was realized that the boundary of the Minkowski sum $\mathcal{M}^{\oplus} \mathcal{M}^{\prime}$ of two bodies $\mathcal{M}$ and $\mathcal{M}^{\prime}$ is a subset of the convolution surface $\partial \mathcal{M} * \partial \mathcal{M}^{\prime}$ of their boundaries $\partial \mathcal{M}$ and $\partial \mathcal{M}$ which is obtained by adding points on $\partial \mathcal{M}$ to points on $\partial \mathcal{M}^{\prime}$ that share the same unit normal vector. When $\mathcal{M}$ and $\mathcal{M}^{\prime}$ are convex, the boundary of $\mathcal{M} \oplus \mathcal{M}^{\prime}$ coincides with $\partial \mathcal{M} * \partial \mathcal{M}^{\prime}$ while, when convexity is not guaranteed, the former set is contained in the latter and can be recovered through a trimming procedure that, roughly speaking, amounts to remove self-intersections and/or internal contours from the convolution surface. As a matter of fact, lot of work has been done to propose numerical algorithms to handle the trimming procedure (see, e.g. [11], [12]).

In this paper we apply the CAGD techniques outlined in Sec. II to compute the excluded area for a pair of nonconvex planar molecules. The choice of a two-dimensional context is twofold. First, it is easier to determine convolution curves rather than convolution surfaces: we should keep in mind that in our context we need to compute the excluded area for any relative orientation of $\mathcal{M}$ and $\mathcal{M}^{\prime}$ and this in general leads to a long distinction of subcases that are more difficult to handle in three-dimensions. Moreover, in the liquid crystal literature there has been recently a surge of interest for two-dimensional systems that can be used to mimic the behaviour of liquid crystals confined between two close, parallel walls. The influence of molecular shapes upon the the orientational transition in an ensemble of two-dimensional hard rod fluids has been stressed in [13] where, using a scaled particle theory, it was proved that changing the length-to-width ratio of hard rectangles makes the isotropic-nematic transition being replaced by a transition in which the orientational order possesses a fourfold symmetry axis. The rich variety of phase transitions in two-dimensional systems has been checked recently by experiments [14] and simulations [15]. Here, in Section III, we illustrate in detail the computations of the excluded area for a family of non-convex molecules that is a direct generalization of the discorectangles studied in [16] where the excluded area was employed to build an interaction potential. Strictly speaking, we study two subfamilies: the former is formed by two-dimensional boomerangs obtained by moving the center of a disk of radius $h$ along a circular arc of radius $R>2 h$ that subtends an angle of amplitude $2 \alpha<\pi$ (see Fig. 1a).

The latter is formed by horseshoes that differ from boomerangs only because $2 \alpha>\pi$ (Fig. 1b). We examine in detail the different expressions of the excluded area that emerge on varying both $\alpha$ and the relative orientation. Although in general the excluded area is a complicated function of the relative orientation and of the geometric parameters $\alpha$ and $\eta:=h / R$, it boils down to a simple expression in the limit when $\eta \ll 1$.

The molecules considered in this paper also lack of central symmetry. This makes them a natural candidate to measure the shape polarity effect recently studied in [17]. Here it was shown that the excluded volume for a pair of equal molecules that fail to be centro-symmetric has its absolute minimum when the molecules are anti-parallel, instead of parallel. As remarked in [17] shape polarity survives in a three-dimensional setting, but it is stronger in two-dimensions. By computing the excluded area for horseshoes we prove that, while lack of convexity can make shape polarity effects more evident, lack of symmetry suffices to promote shape polarity in steric interactions. In fact, we explore horseshoes mainly to illustrate the relative importance that lack of central symmetry and lack of convexity have on shape polarity.

The main results of the technical computations shown in Section III are discussed in Section IV. As envisaged in [17], the existence of polar steric interactions could promote the formation of an antiferromorphic smectic phase like $\mathrm{CP}_{A}$ or $\mathrm{SP}_{A}$ phases reproduced, for instance, in computer simulations [18] where the contribution of steric interaction was recognized as crucial. The explicit formulae for the excluded area reported here, in particular those for slender molecules, could be the starting point to build a two-dimensional Onsager model in the spirit of [16]. A conclusive section and a technical appendix containing some ancillary computations close the paper.

\section{EXCLUDED VOLUME, MINKOWSKI SUMS, AND CONVOLUTIONS OF GEOMETRIC OBJECTS}

In this section we collect the definitions and the results employed in the paper to obtain analytic expressions for the excluded area for a pair of congruent, non-convex molecules. We start by defining the Minkowski sum of two sets $A$ and $B$.

Definition II.1 Given two sets $A$ and $B$ belonging to a three-dimensional vector space, their Minkowski sum is the set

$$
A \oplus B:=\{\mathbf{a}+\mathbf{b} \mid \mathbf{a} \in A \text { and } \mathbf{b} \in B\} .
$$

Minkowski sums can be generalized to vector spaces of arbitrary dimension $n$. To visualize the Minkowski sum of $A$ and $B$, the following kinematic construction can be of some help. Place the reference point $O^{\prime}$ of $B$ on a point of the boundary $\partial A$ of $A$ and translate $B$ by keeping $O^{\prime}$ along $\partial A$ : the contour of the region spanned by $B$ during this 
motion is the boundary of $A \oplus B$. There is an intimate relation between the Minkowski addition of two sets and their excluded region $\mathcal{E}(A, B)$ that can be formalized by the following theorem [7]

Theorem II.1 Let $A$ and $B$ two sets, and let

$$
\ominus B:=\{\mathbf{b} \mid-\mathbf{b} \in B\}
$$

be the Minkowski inverse of $B$ with respect to its reference point $O^{\prime}$. The set $\mathcal{E}(A, B)$ is obtained by a translation of $A \ominus B:=A \oplus(\ominus B)$ and, in particular,

$$
V[\mathcal{E}(A, B)]=V(A \ominus B)
$$

where $V(A \ominus B)$ is the volume bounded by the set $A \ominus B$, called the Minkowski difference of $A$ and $B$.

The importance of this theorem in excluded-volume computations was stressed only recently by Mulder in [7], to which we refer the reader for a proof. It should be noted, however, that this result has been known for a long time in the community of CAGD. In particular, a characterization of the excluded region for sets in $\mathbf{R}^{2}$ in terms of their Minkowski sum appeared in [10] and [19]. It is important to note that Theorem II.1 is not restricted to convex bodies.

However, when one of the bodies fails to be convex, the explicit determination of $\partial(A \oplus B)$ becomes rather intricate and, in fact, there is no general analytic procedure to obtain it. Despite of this, the connection between the Minkowski sum of two sets and their convolution surface can be exploited. Given two sets $A$ and $B$, let $\boldsymbol{\nu}_{A}$ and $\boldsymbol{\nu}_{B}$ denote the fields of the outer unit normals of $\partial A$ and $\partial B$. Then, the convolution surface $\partial A * \partial B$ of $\partial A$ and $\partial B$ is defined as the set $[20]$

$$
\partial A * \partial B:=\left\{\mathbf{a}+\mathbf{b} \mid \mathbf{a} \in \partial A, \mathbf{b} \in \partial B, \boldsymbol{\nu}_{A}(\mathbf{a})=\boldsymbol{\nu}_{B}(\mathbf{b})\right\} .
$$

In two space-dimensions we can define the convolution curve of two curves $A$ and $B$ in a completely analogous way $[9,19]$. If the sets $A$ and $B$ are convex, $\partial(A \oplus B)$ coincides with $\partial A * \partial B$, but in general we can only say that

$$
\partial(A \oplus B) \subseteq \partial A * \partial B
$$

since the convolution set can have internal boundaries and self-intersections that do not appear in the Minkowski sum. Hence, as soon as the convolution set of $A$ and $B$ has been obtained, a trimming procedure is needed to remove the spurious arcs that do not enter the set $\partial(A \oplus B)$. It is precisely this trimming procedure which is difficult to implement analytically while, on the other hand, several numerical algorithms have been proposed (see, e.g. [11], $[12])$.

Given a point a on a closed surface $\mathcal{S}$ or on a closed curve $\mathcal{C}$, the map $\mathcal{N}: \mathbf{a} \mapsto \boldsymbol{\nu}(\mathbf{a})$ is the Gauss map of $\mathcal{S}$ or $\mathcal{C}$ at $\mathbf{a}$ and so, by equation (II.2), to obtain the convolution set we need to identify pairs of points on $\partial A$ and $\partial B$ at which the Gauss maps attain one and the same value. Two patches $\mathcal{P}_{A}$ and $\mathcal{P}_{B}$ belonging to $\partial A$ and $\partial B$, respectively, are said to be compatible [9] if there is a bijective correspondence that assign to each point $\mathbf{a} \in \mathcal{P}_{A}$ a point $\mathbf{b} \in \mathcal{P}_{B}$ such that $\mathcal{N}(\mathbf{a})=\mathcal{N}(\mathbf{b})$. Hence, compatible patches will contribute to the set $\partial A * \partial B$. In two space dimensions, let $P \in \partial A$ and $Q \in \partial B$ be two points on compatible $\operatorname{arcs} \mathcal{C}_{i} \subset \partial A$ and $\mathcal{K}_{j} \subset \partial B$ at which $\mathcal{N}(P)=\mathcal{N}(Q)$. If $\partial A$ and $\partial B$ are parameterized by $t$ and $\tau$, respectively, and $t_{0}$ is the value of $t$ at $P$, the value of $\tau$ at $Q$ can be written as $\tau=\tau\left(t_{0}\right)$. Then, the point

$$
(P * Q)\left(t_{0}\right):=P\left(t_{0}\right)-O+Q\left(\tau\left(t_{0}\right)\right)-O
$$

belongs to the convolution curve $\partial A * \partial B$. Building the map $t \mapsto \tau(t)$ requires a knowledge of the curvature of both $\partial A$ and $\partial B$. However, in the problem we are concerned with the compatible arcs are circles and so $\tau(t)$ is simply a shift: $\tau(t)=t+t_{0}$. The convolution curve has been parameterized by the same parameter $t$ used for $\partial A$ but, clearly, one could work with the inverse map $\tau \mapsto \tau(t)$ and parameterize the convolution curve in terms of $\tau$. The curve that shares the same parameter with the convolution curve is called the base curve. The choice of the base curve is a matter of convenience, and it can vary when different pairs of compatible arcs are considered.

After these remarks, we outline the procedure to compute the excluded area for two-dimensional molecules $\mathcal{M}$ and $\mathcal{M}^{\prime}$ in four steps.

1. For a given relative orientation, we identify the $\operatorname{arcs}$ on $\partial \mathcal{M}$ and $\partial \mathcal{M}^{\prime}$ that share the same Gauss map.

2. Joining together the arcs found at step 1, we build the convolution curve $\partial \mathcal{M} * \partial \mathcal{M}^{\prime}$.

3. We remove from $\partial \mathcal{M} * \partial \mathcal{M}^{\prime}$ both internal contours and self-intersections to obtain the set $\partial\left(\mathcal{M} \oplus \mathcal{M}^{\prime}\right)$.

4. We compute the area enclosed by $\partial\left(\mathcal{M} \oplus \mathcal{M}^{\prime}\right)$ which, by Theorem II.1, is the excluded area of $\mathcal{M}$ and $\ominus \mathcal{M}^{\prime}$.

In the following sections we apply this program to a pair of two-dimensional boomerangs or horseshoes as those shown in Fig. 1. 


\section{BOOMERANGS AND HORSESHOES}

In the $\left\{\boldsymbol{e}_{x}, \boldsymbol{e}_{y}\right\}$-plane we consider a circular arc $A_{0} B_{0}$ of radius $R$, centered at a point $O$ and subtending an angle $2 \alpha$ (Fig. 1). We introduce a reference molecule $\mathcal{M}_{0}$ bounded by: $a$ ) two concentric circular arcs $\mathcal{C}_{1}^{0}$ and $\mathcal{C}_{3}^{0}$ centered at $O$, with radius $R-h$ and $R+h$, both subtending an angle $2 \alpha$ and $b$ ) two semicircles $\mathcal{C}_{2}^{0}$ and $\mathcal{C}_{4}^{0}$ of radius $h$, centered at $A_{0}$ and $B_{0}$, respectively: see Fig. 2 .

We first determine the maximum value $\alpha_{M}$ of $\alpha$ compatible with the absence of self-intersections in the profile of $\mathcal{M}_{0}$. This is easily obtained by imposing that two circles of radius $h$ centered at $A_{0}=(-R \sin \alpha, R \cos \alpha)$ and $B_{0}=(R \sin \alpha, R \cos \alpha)$ are mutually tangent. A straightforward computation shows that this occurs when

$$
\alpha=\alpha_{M}:=\pi-\arcsin (\sqrt{\eta}),
$$

where we have introduced the dimensionless parameter

$$
\eta:=\frac{h}{R}
$$

that ranges the interval $\left(0, \frac{1}{2}\right)$. Hereafter, we rescale lengths to $R$.

To exploit the symmetry with respect to molecules' exchange, it is expedient to consider $\mathcal{M}$ and $\mathcal{M}^{\prime}$ as images of $\mathcal{M}_{0}$ under two rotations $\mathbf{Q}\left(\boldsymbol{e}_{z}, \chi\right)$ by suitable angles $\chi$ about the $\boldsymbol{e}_{z}:=\boldsymbol{e}_{x} \times \boldsymbol{e}_{y}$ axis passing through $O$ : precisely, $\mathcal{M}$ is the image of $\mathcal{M}_{0}$ under $\mathbf{Q}\left(\boldsymbol{e}_{z},-\vartheta / 2\right)$ and $\mathcal{M}^{\prime}$ is the image of $\mathcal{M}_{0}$ under $\mathbf{Q}\left(\boldsymbol{e}_{z}, \vartheta / 2\right)$. Hence, $\vartheta \in[0, \pi]$ is the relative angle between the molecules $\mathcal{M}$ and $\mathcal{M}^{\prime}$. By orienting $\mathcal{M}$ counterclockwise, its arcs $\mathcal{C}_{i}$ can be parameterized as

$$
\begin{gathered}
\mathcal{C}_{1}: \quad(P-O)(t)=(1+\eta)\left[-\sin \left(t-\alpha-\frac{\vartheta}{2}\right) \boldsymbol{e}_{x}+\cos \left(t-\alpha-\frac{\vartheta}{2}\right) \boldsymbol{e}_{y}\right], \quad t \in[0,2 \alpha] \\
\mathcal{C}_{2}: \quad(P-A)(t)=\eta\left[-\sin \left(t+\alpha-\frac{\vartheta}{2}\right) \boldsymbol{e}_{x}+\cos \left(t+\alpha-\frac{\vartheta}{2}\right) \boldsymbol{e}_{y}\right], \quad t \in[0, \pi] \\
\mathcal{C}_{3}: \quad(P-O)(t)=(1-\eta)\left[-\sin \left(-t+\alpha-\frac{\vartheta}{2}\right) \boldsymbol{e}_{x}+\cos \left(-t+\alpha-\frac{\vartheta}{2}\right) \boldsymbol{e}_{y}\right], \quad t \in[0,2 \alpha] \\
\mathcal{C}_{4}: \quad(P-B)(t)=\eta\left[-\sin \left(\pi+t-\alpha-\frac{\vartheta}{2}\right) \boldsymbol{e}_{x}+\cos \left(\pi+t-\alpha-\frac{\vartheta}{2}\right) \boldsymbol{e}_{y}\right], \quad t \in[0, \pi],
\end{gathered}
$$

where $A-O, B-O$, and the $\operatorname{arcs} \mathcal{C}_{i}$ are the images of $A_{0}-O, B_{0}-O$ and the $\operatorname{arcs} \mathcal{C}_{i}^{0}$ under $\mathbf{Q}\left(\boldsymbol{e}_{z},-\frac{\vartheta}{2}\right)$. We note that when $\mathcal{M}_{0}$ is oriented counterclockwise, the outer unit normal vectors of $\mathcal{C}_{i}^{0}$ rotate counterclockwise along the convex arcs but clockwise along the concave arc (see Fig. 3).

The arguments of the trigonometric functions in equations (III.2-III.5) might look awkward. We chose the keep one and the same format $-\sin (\cdot) \boldsymbol{e}_{x}+\cos (\cdot) \boldsymbol{e}_{y}$ to make comparisons between the Gauss maps of different arcs easier. Similarly, we parameterize the arcs forming the molecule $\mathcal{M}^{\prime}$ as

$$
\begin{aligned}
& \mathcal{K}_{1}: \quad(P-O)(t)=(1+\eta)\left[-\sin \left(t-\alpha+\frac{\vartheta}{2}\right) \boldsymbol{e}_{x}+\cos \left(t-\alpha+\frac{\vartheta}{2}\right) \boldsymbol{e}_{y}\right], \quad t \in[0,2 \alpha] \\
& \mathcal{K}_{2}: \quad\left(P-A^{\prime}\right)(t)=\eta\left[-\sin \left(t+\alpha+\frac{\vartheta}{2}\right) \boldsymbol{e}_{x}+\cos \left(t+\alpha+\frac{\vartheta}{2}\right) \boldsymbol{e}_{y}\right], \quad t \in[0, \pi] \\
& \mathcal{K}_{3}: \quad(P-O)(t)=(1-\eta)\left[-\sin \left(-t+\alpha+\frac{\vartheta}{2}\right) \boldsymbol{e}_{x}+\cos \left(-t+\alpha+\frac{\vartheta}{2}\right) \boldsymbol{e}_{y}\right], \quad t \in[0,2 \alpha] \\
& \mathcal{K}_{4}: \quad\left(P-B^{\prime}\right)(t)=\eta\left[-\sin \left(\pi+t-\alpha+\frac{\vartheta}{2}\right) \boldsymbol{e}_{x}+\cos \left(\pi+t-\alpha+\frac{\vartheta}{2}\right) \boldsymbol{e}_{y}\right], \quad t \in[0, \pi]
\end{aligned}
$$

where $A^{\prime}-O, B^{\prime}-O$, and the $\operatorname{arcs} \mathcal{K}_{i}$ are the images of $A_{0}-O, B_{0}-O$ and the $\operatorname{arcs} \mathcal{C}_{i}^{0}$ under $\mathbf{Q}\left(\boldsymbol{e}_{z}, \frac{\vartheta}{2}\right)$. Clearly,

$$
\begin{array}{ll}
A-O=\left[-\sin \left(\alpha-\frac{\vartheta}{2}\right) \boldsymbol{e}_{x}+\cos \left(\alpha-\frac{\vartheta}{2}\right) \boldsymbol{e}_{y}\right] & B-O=\left[\sin \left(\alpha+\frac{\vartheta}{2}\right) \boldsymbol{e}_{x}+\cos \left(\alpha+\frac{\vartheta}{2}\right) \boldsymbol{e}_{y}\right] \\
A^{\prime}-O=\left[-\sin \left(\alpha+\frac{\vartheta}{2}\right) \boldsymbol{e}_{x}+\cos \left(\alpha+\frac{\vartheta}{2}\right) \boldsymbol{e}_{y}\right] & B^{\prime}-O=\left[\sin \left(\alpha-\frac{\vartheta}{2}\right) \boldsymbol{e}_{x}+\cos \left(\alpha-\frac{\vartheta}{2}\right) \boldsymbol{e}_{y}\right] .
\end{array}
$$


We now determine the convolution curve $\partial \mathcal{M} * \partial \mathcal{M}^{\prime}$ of $\partial \mathcal{M}=\cup_{i=1}^{4} \mathcal{C}_{i}$ and $\partial \mathcal{M}^{\prime}=\cup_{i=1}^{4} \mathcal{K}_{i}$ from which we will extract information on the boundary of the Minkowski sum $\partial\left(\mathcal{M} \oplus \mathcal{M}^{\prime}\right)$ and, then, on the excluded area between $\mathcal{M}$ and $\ominus \mathcal{M}^{\prime}$.

To understand our procedure, we focus on the case $\alpha \in\left[0, \frac{\pi}{4}\right]$ and $\vartheta \in[0,2 \alpha]$. The crosses in Fig. 4 illustrate the Gauss maps on the arcs of two molecules rotated by an angle $\vartheta$.

The sets $\mathcal{N}\left(\mathcal{C}_{1}\right), \ldots, \mathcal{N}\left(\mathcal{C}_{4}\right)$ are the angles $\angle V_{1} O V_{2}, \angle V_{2} O V_{4}, \angle V_{4} O V_{3}$, and $\angle V_{1} O V_{3}$, respectively. On the other hand, the sets $\mathcal{N}\left(\mathcal{K}_{1}\right), \ldots, \mathcal{N}\left(\mathcal{K}_{4}\right)$ are the angles $\angle V_{1}^{\prime} O V_{2}^{\prime}, \angle V_{2}^{\prime} O V_{4}^{\prime}, \angle V_{4}^{\prime} O V_{3}^{\prime}$, and $\angle V_{1}^{\prime} O V_{3}^{\prime}$, respectively. When $\vartheta \in[0,2 \alpha]$ the compatible set of $\mathcal{C}_{1}$ and $\mathcal{K}_{1}$ is the arc $\angle V_{1}^{\prime} O V_{2}$ of amplitude $2 \alpha-\vartheta$. Using $\mathcal{K}_{1}$ as a base curve, $t \in[0,2 \alpha-\vartheta]$ along the compatible set. Similarly, the compatible set of $\mathcal{C}_{2}$ and $\mathcal{K}_{1}$ is the arc $\angle V_{2} O V_{2}^{\prime}$ of amplitude $\vartheta$ along which $t \in[0, \vartheta]$, if $\mathcal{C}_{2}$ is taken as the base curve. By repeating such comparisons for all possible pairs of arcs, the convolution curve can be retraced directly.

As a fact, the sets that enter the convolution curve depend on the values of $\alpha \in\left[0, \alpha_{M}\right]$ and $\vartheta \in[0, \pi]$. We distinguish four cases, according to whether $\alpha \in\left[0, \frac{\pi}{4}\right], \alpha \in\left[\frac{\pi}{4}, \frac{\pi}{2}\right], \alpha \in\left[\frac{\pi}{2}, \frac{3 \pi}{4}\right]$ and $\alpha \in\left[\frac{3 \pi}{4}, \alpha_{M}\right]$.

\section{A. Boomerangs I: $\alpha \in\left[0, \frac{\pi}{4}\right]$}

In this case, the following regimes exist, according to the value of $\vartheta: \vartheta \in[0,2 \alpha], \vartheta \in[2 \alpha, \pi-2 \alpha], \vartheta \in[\pi-2 \alpha, \pi]$ : the last interval will be split into three subintervals, as explained later.

$$
\text { 1. } \vartheta \in[0,2 \alpha]
$$

We list here the arcs that contribute to the convolution curve $\partial \mathcal{M} * \partial \mathcal{M}^{\prime}$, obtained through the procedure sketched in Fig. 4. We denote the compatible set $\mathcal{C}_{i} * \mathcal{K}_{j}$ of $\mathcal{C}_{i}$ and $\mathcal{K}_{j}$ as $C_{i j}$ : in general $C_{i j} \neq C_{j i}$, if $i \neq j$. Since the molecules are formed only by circular arcs, $C_{i j}$ are circular arcs too. The second and the third column in the subsequent tables contain the radii of $C_{i j}$, scaled to $R$, and their centers. The fourth and the fifth columns show the values of $t$ along $C_{i j}$ and the base curve chosen to parameterize it. The choice of the base curve was made according to the following criteria: when a concave arc is involved, the base curve always coincides with the convex segment; if no concave segment is involved in $C_{i j}$, the base curve is the arc that allows parameterizing $C_{i j}$ with $t$ starting from 0 : this criterion has already been followed in the example of Fig. 4. The explicit representation of the arcs $C_{i j}$ is given in Appendix A.

\begin{tabular}{|l|l|l|l|l|}
\hline arc & radius & center & $t$ & base curve \\
\hline$C_{11}$ & $2(1+\eta)$ & $O$ & {$[0,2 \alpha-\vartheta]$} & $\mathcal{K}_{1}(t)$ \\
$C_{14}$ & $1+2 \eta$ & $B^{\prime}$ & {$[0, \vartheta]$} & $\mathcal{C}_{1}(t)$ \\
$C_{21}$ & $1+2 \eta$ & $A$ & {$[0, \vartheta]$} & $\mathcal{C}_{2}(t)$ \\
$C_{22}$ & $2 \eta$ & $A+A^{\prime}$ & {$[0, \pi-\vartheta]$} & $\mathcal{K}_{2}(t)$ \\
$C_{23}$ & $2 \eta-1$ & $A$ & {$[\pi-2 \alpha+\vartheta, \pi]$} & $\mathcal{C}_{2}(t)$ \\
$C_{24}$ & $2 \eta$ & $A+B^{\prime}$ & {$[0,2 \alpha-\vartheta]$} & $\mathcal{K}_{4}(t)$ \\
$C_{32}$ & $2 \eta-1$ & $A^{\prime}$ & {$[0,2 \alpha]$} & $\mathcal{K}_{2}(t)$ \\
$C_{34}$ & $2 \eta-1$ & $B^{\prime}$ & {$[0,2 \alpha-\vartheta]$} & $\mathcal{K}_{4}(t)$ \\
$C_{42}$ & $2 \eta$ & $B+A^{\prime}$ & {$[0,2 \alpha+\vartheta]$} & $\mathcal{C}_{4}(t)$ \\
$C_{43}$ & $2 \eta-1$ & $B$ & {$[\vartheta, 2 \alpha+\vartheta]$} & $\mathcal{C}_{4}(t)$ \\
$C_{44}$ & $2 \eta$ & $B+B^{\prime}$ & {$[0, \pi-\vartheta]$} & $\mathcal{K}_{4}(t)$ \\
\hline
\end{tabular}

Arcs like $C_{13}, C_{31}$ and $C_{33}$ are excluded because either the concave segment has larger curvature than the convex one $\left(C_{13}, C_{31}\right)$ or both the segments are concave $\left(C_{33}\right)$. Hence, as proved in [9], they cannot contribute to the boundary of the Minkowski sum $\partial\left(\mathcal{M} \oplus \mathcal{M}^{\prime}\right)$. We note that this property is peculiar of the two-dimensional setting and cannot be extended to three-dimensional problems. In the present case, the segments $C_{23}, C_{24}$ and $C_{34}$ should be trimmed since they are inner contours of the convolution curve and so cannot contribute to $\partial\left(\mathcal{M} \oplus \mathcal{M}^{\prime}\right)$. Finally, to account correctly for the convexity of the arcs $C_{3 j}$ and $C_{i 3}$ we have formally considered the radii of $\mathcal{C}_{3}$ and $\mathcal{K}_{3}$ as negative. The coordinate system we chose makes it possible to show that the pairs $\left(C_{11}, C_{42}\right),\left(C_{14}, C_{21}\right),\left(C_{44}, C_{22}\right)$, and $\left(C_{43}, C_{32}\right)$ are symmetric with respect to the $y$-axis. This is indeed a general property that does not depend on the specific values of $\alpha$ or $\vartheta$ and that permits focussing attention on the reduced boundary $\mathcal{R}_{\oplus}$ defined as the subset of $\partial\left(\mathcal{M}_{\oplus} \oplus \mathcal{M}^{\prime}\right)$ where $x \geq 0$. Clearly, to obtain $\partial\left(\mathcal{M} \oplus \mathcal{M}^{\prime}\right)$ we paste $\mathcal{R}_{\oplus}$ and its symmetric image with respect to the $y$-axis. 
The area $A$ enclosed by the set $\partial\left(\mathcal{M} \oplus \mathcal{M}^{\prime}\right)$ is a function $A=A(\alpha, \vartheta, R, h)$ and we compute its dimensionless counterpart $A(\alpha, \vartheta, \eta):=A(\alpha, \vartheta, R, h) / R^{2}$. By Theorem II.1, $A(\alpha, \vartheta, \eta)$ is the excluded area for $\mathcal{M}$ and $\mathcal{M}^{\prime}$ when their relative orientation is $\pi-\vartheta$. To compute $A(\alpha, \vartheta, \eta)$, we decompose the reduced boundary $\mathcal{R}_{\oplus}$ as shown in Figure 5.

Since the area bounded by a circular segment of radius $r$ subtending an angle $\beta$ is

$$
\operatorname{Sett}(r, \beta)=\frac{r^{2}}{2}[\beta-\sin \beta]
$$

and the area of a trapezium of height $h_{i}$ and bases $b_{i}, b_{i+1}$ is

$$
\mathcal{T}\left(h_{i}, b_{i}, b_{i+1}\right)=\frac{h_{i}}{2}\left(b_{i}+b_{i+1}\right)
$$

we conclude that

$$
\begin{aligned}
A(\alpha, \vartheta, \eta)= & 2\left[\frac{1}{2} \operatorname{Sett}(2(\eta+1), 2 \alpha-\vartheta)+\operatorname{Sett}(1+2 \eta, \vartheta)+\operatorname{Sett}(2 \eta, \pi-\vartheta)-\operatorname{Sett}(2 \eta-1,2 \alpha)+\frac{1}{2} \operatorname{Sett}(2 \eta, 2 \alpha+\vartheta)\right. \\
& \left.+\mathcal{T}\left(h_{1}, b_{1}, b_{2}\right)+\mathcal{T}\left(h_{2}, b_{2}, b_{3}\right)+\mathcal{T}\left(h_{3}, b_{3}, b_{4}\right)\right],
\end{aligned}
$$

where the minus sign in front of $\operatorname{Sett}(2 \eta-1,2 \alpha)$ accounts for the concavity of the segment $C_{43}$. Finally, by setting $x_{i j}:=\left(C_{i j}-O\right) \cdot \boldsymbol{e}_{x}$ and $y_{i j}:=\left(C_{i j}-O\right) \cdot \boldsymbol{e}_{y}$ we also have

$$
b_{1}=x_{14}(\vartheta) \quad b_{2}=x_{14}(0) \quad b_{3}=x_{44}(0) \quad b_{4}=x_{43}(\vartheta+2 \alpha)
$$

and

$$
h_{1}=y_{14}(\vartheta)-y_{14}(0) \quad h_{2}=y_{44}(\pi-\vartheta)-y_{44}(0) \quad h_{3}=y_{43}(\vartheta)-y_{43}(\vartheta+2 \alpha)
$$

that can be computed by using the results of Appendix A. Then, it is not difficult to prove that

$$
A(\alpha, \vartheta, \eta)=2 \alpha-\vartheta+2 \sin \vartheta-\sin (2 \alpha+\vartheta)+16 \alpha \eta+4 \pi \eta^{2} .
$$

$$
\text { 2. } \vartheta \in[2 \alpha, \pi-2 \alpha]
$$

Figure $5 b$ ) shows the set $\mathcal{R}_{\oplus}$ when $\vartheta$ ranges in $[2 \alpha, \pi-2 \alpha]$. In the following table, we list the basic properties of the arcs that enter $\mathcal{R}_{\oplus}$ (see also Appendix A for details)

\begin{tabular}{|l|l|l|l|l|}
\hline arc & radius & center & $t$ & base curve \\
\hline$C_{24}$ & $2 \eta$ & $A+B^{\prime}$ & {$[0, \vartheta / 2-\alpha]$} & $\mathcal{C}_{2}(t)$ \\
$C_{14}$ & $1+2 \eta$ & $B^{\prime}$ & {$[0,2 \alpha]$} & $\mathcal{C}_{1}(t)$ \\
$C_{44}$ & $2 \eta$ & $B+B^{\prime}$ & {$[0, \pi-\vartheta]$} & $\mathcal{K}_{4}(t)$ \\
$C_{43}$ & $2 \eta-1$ & $B$ & {$[\vartheta, \vartheta+2 \alpha]$} & $\mathcal{C}_{4}(t)$ \\
$C_{42}$ & $2 \eta$ & $B+A^{\prime}$ & {$[\alpha+\vartheta / 2,2 \alpha+\vartheta]$} & $\mathcal{C}_{4}(t)$ \\
\hline
\end{tabular}

The area bounded by the set $\partial\left(\mathcal{M} \oplus \mathcal{M}^{\prime}\right)$ is

$$
\begin{aligned}
A(\alpha, \vartheta, \eta)= & 2\left[\frac{1}{2} \operatorname{Sett}(2 \eta, \vartheta-2 \alpha)+\operatorname{Sett}(1+2 \eta, 2 \alpha)+\operatorname{Sett}(2 \eta, \pi-\vartheta)-\operatorname{Sett}(2 \eta-1,2 \alpha)+\frac{1}{2} \operatorname{Sett}(2 \eta, 2 \alpha+\vartheta)\right. \\
& \left.+\mathcal{T}\left(h_{1}^{\prime}, b_{1}^{\prime}, b_{2}\right)+\mathcal{T}\left(h_{2}, b_{2}, b_{3}\right)+\mathcal{T}\left(h_{3}, b_{3}, b_{4}\right)\right]
\end{aligned}
$$

where now

$$
b_{1}^{\prime}=x_{14}(2 \alpha) \quad h_{1}^{\prime}=y_{14}(2 \alpha)-y_{14}(0)
$$

while $b_{2}-b_{4}$ and $h_{2}, h_{3}$ are still given by equations (III.14) and (III.15). With the results shown in Appendix A we obtain

$$
A(\alpha, \vartheta, \eta)=4 \sin \vartheta \sin ^{2} \alpha+16 \alpha \eta+4 \pi \eta^{2} .
$$

In the limit where $R \rightarrow \infty$ and $\alpha \rightarrow 0$ in such a way that $\alpha R \rightarrow L / 2$, the molecules considered here approach a pair of equal discorectangles studied in Ref. [16]. In this limit, (III.19) is the only expression of the excluded area and, in dimensional units, it tends to

$$
4 \sin \vartheta(R \sin \alpha)^{2}+16 \alpha h R+4 \pi h^{2}=8 h L+4 \pi h^{2}+L^{2} \sin \vartheta
$$

which is precisely equation (4) of [16] when we set $2 h=\sigma=\sigma_{s}$ and $L=L_{s}$. 
where now

When $\vartheta>\pi-2 \alpha$, computations are more intricate since the end-points of some admissible $\operatorname{arcs} C_{i j}$ of $\mathcal{R}_{\oplus}$ lie in the interior of $\partial\left(\mathcal{M} \oplus \mathcal{M}^{\prime}\right)$, and so the intersections between different arcs $C_{i j}$ are to be computed. It turns out that the set $\vartheta \in[\pi-2 \alpha, \pi]$ has to be split into three subsets according to whether $\vartheta \in\left[\pi-2 \alpha, \vartheta_{1243}\right], \vartheta \in\left[\vartheta_{1243}, \vartheta_{1244}\right]$ or $\vartheta \in\left[\vartheta_{1244}, \pi\right]$, where the values $\vartheta_{1243}$ and $\vartheta_{1244}$ are defined as follows. By using the procedure sketched in Fig. 4 the arc $C_{12}$ could contribute to $\mathcal{R}_{\oplus}$ when $t \in[0, \vartheta-\pi+2 \alpha]$. However, (see Fig. 6) $C_{12}$ intersects $C_{43}$ at a value $t_{1243}$ of $t \in(0, \vartheta-\pi+2 \alpha)$ until the critical value $\vartheta_{1243}(\alpha, \eta)$ of $\vartheta$ is attained, at which the arc $C_{43}$ lies completely in the interior of $\mathcal{R}_{\oplus}$. From this value, $C_{12}$ intersects the arc $C_{44}$ until $\vartheta$ attains the value $\vartheta_{1244}(\alpha, \eta)>\vartheta_{1243}(\alpha, \eta)$. When $\vartheta \in\left[\vartheta_{1244}, \pi\right]$, also $C_{44}$ lies in the interior of $\mathcal{R}_{\oplus}$ and $C_{12}$ intersects the arc $C_{14}$. In the following three tables we summarize the structure of $\mathcal{R}_{\oplus}$ when $\vartheta \in\left[\pi-2 \alpha, \vartheta_{1243}\right], \vartheta \in\left[\vartheta_{1243}, \vartheta_{1244}\right]$, and $\vartheta \in\left[\vartheta_{1244}, \pi\right]$, respectively. The explicit computations of these critical values of $\vartheta$ as well as of the limits $t_{1243}, t_{4312}, t_{1244}, t_{4412}, t_{1214}, t_{1412}$ are shown in Appendix A: see equations (A.5), (A.6), (A.11), (A.12), (A.15), and (A.16).

\begin{tabular}{|l|l|l|l|l|}
\hline arc & radius & center & $t$ & base curve \\
\hline$C_{24}$ & $2 \eta$ & $A+B^{\prime}$ & {$[0, \vartheta / 2-\alpha]$} & $\mathcal{C}_{2}(t)$ \\
$C_{14}$ & $1+2 \eta$ & $B^{\prime}$ & {$[0,2 \alpha]$} & $\mathcal{C}_{1}(t)$ \\
$C_{44}$ & $2 \eta$ & $B+B^{\prime}$ & {$[0, \pi-\vartheta]$} & $\mathcal{K}_{4}(t)$ \\
$C_{43}$ & $2 \eta-1$ & $B$ & {$\left[\vartheta, t_{4312}\right]$} & $\mathcal{C}_{4}(t)$ \\
$C_{12}$ & $1+2 \eta$ & $A^{\prime}$ & {$\left[0, t_{1243}\right]$} & $\mathcal{C}_{1}(t)$ \\
$C_{42}$ & $2 \eta$ & $A^{\prime}+B$ & {$\left[\pi-\frac{1}{2}(\vartheta+2 \alpha), 2 \pi-(\vartheta+2 \alpha)\right]$} & $\mathcal{K}_{2}(t)$ \\
\hline
\end{tabular}

The area enclosed by the set $\partial\left(\mathcal{M} \oplus \mathcal{M}^{\prime}\right)$ is given by

$$
\begin{aligned}
A(\alpha, \vartheta, \eta)= & 2\left[\frac{1}{2} \operatorname{Sett}(2 \eta, \vartheta-2 \alpha)+\operatorname{Sett}(1+2 \eta, 2 \alpha)+\operatorname{Sett}(2 \eta, \pi-\vartheta)-\operatorname{Sett}\left(2 \eta-1, t_{4312}-\vartheta\right)+\operatorname{Sett}\left(1+2 \eta, t_{1243}\right)\right. \\
& \left.+\frac{1}{2} \operatorname{Sett}(2 \eta, 2 \pi-(2 \alpha+\vartheta))+\mathcal{T}\left(h_{1}^{\prime}, b_{1}^{\prime}, b_{2}\right)+\mathcal{T}\left(h_{2}, b_{2}, b_{3}\right)+\mathcal{T}\left(h_{3}^{\prime}, b_{3}, b_{4}^{\prime}\right)+\mathcal{T}\left(h_{4}, b_{4}^{\prime}, b_{5}\right)\right]
\end{aligned}
$$

$$
b_{4}^{\prime}=x_{43}\left(t_{4312}\right) \quad b_{5}=x_{12}(0) \quad h_{3}^{\prime}=y_{43}(\vartheta)-y_{43}\left(t_{4312}\right) \quad h_{4}=y_{12}\left(t_{1243}\right)-y_{12}(0) .
$$

The explicit expression of the excluded area could be written but it would not be very telling. When attention is restricted to the case $\eta \ll 1$, the approximate excluded area takes the form

$$
A(\alpha, \vartheta, \eta)=2(\vartheta+2 \alpha-\pi)+2 \sin \vartheta+2 \cos \vartheta \sin 2 \alpha+16 \alpha \eta+O\left(\eta^{2}\right) .
$$

$$
\text { 4. } \vartheta \in\left[\vartheta_{4312}, \vartheta_{4412}\right]
$$

When $\vartheta$ exceeds the value $\vartheta_{4312}$, the arc $C_{43}$ lies completely in the interior of $\mathcal{R}_{\oplus}$ and the arc $\mathcal{C}_{12}$ intersects the arc $C_{44}$ as shown in Fig. $7 a$ ).

The admissible values of $t$ along $\mathcal{C}_{44}$ range the interval [0, $\pi-\vartheta-t_{4412}$ ] while along $\mathcal{C}_{12} t$ ranges the interval [0, $\left.t_{1244}\right]$, where the values of $t_{1244}$ and $t_{4412}$ are given in equations (A.11) and (A.12) of Appendix A. The excluded area is now given by

$$
\begin{aligned}
A(\alpha, \vartheta, \eta)= & 2\left[\frac{1}{2} \operatorname{Sett}(2 \eta, \vartheta-2 \alpha)+\operatorname{Sett}(1+2 \eta, 2 \alpha)+\operatorname{Sett}\left(2 \eta, \pi-\vartheta-t_{4412}\right)+\operatorname{Sett}\left(1+2 \eta, t_{1244}\right)\right. \\
& \left.+\frac{1}{2} \operatorname{Sett}(2 \eta, 2 \pi-(2 \alpha+\vartheta))+\mathcal{T}\left(h_{1}^{\prime}, b_{1}^{\prime}, b_{2}\right)+\mathcal{T}\left(h_{2}^{\prime}, b_{2}, b_{3}^{\prime}\right)+\mathcal{T}\left(h_{3}^{\prime}, b_{3}^{\prime}, b_{5}\right)\right]
\end{aligned}
$$

where

$$
b_{3}^{\prime}=x_{44}\left(t_{4412}\right) \quad h_{2}^{\prime}=y_{44}(\pi-\vartheta)-y_{44}\left(t_{4412}\right) \quad h_{3}^{\prime \prime}=y_{12}\left(t_{1244}\right)-y_{12}(0) .
$$

As discussed in the Appendix, when $\eta \rightarrow 0$ and $\alpha<\frac{\pi}{2}$, then

$$
\vartheta_{4312} \simeq \pi-2 \eta \tan \alpha+O\left(\eta^{2}\right) \quad \vartheta_{4412} \simeq \pi-2 \eta \tan \alpha+O\left(\eta^{2}\right)
$$

so that, if we keep only the linear terms in $\eta, \vartheta_{4312}$ and $\vartheta_{4412}$ coincide and this interval can be skipped. For future use, we define the angle

$$
\vartheta_{a}(\alpha, \eta):=\pi-2 \eta \tan \alpha
$$

Clearly this analysis is valid only if $\alpha$ is not close to $\frac{\pi}{2}$. In fact, when $\alpha>\frac{\pi}{2}$ and $\eta \rightarrow 0$ the angles $\vartheta_{4312}$ and $\vartheta_{4412}$ approach the limit value $2(\pi-\alpha)$ and so at $\alpha=\frac{\pi}{2} \vartheta_{4312}$ and $\vartheta_{4412}$ have a knee where they depart from $\pi$ like $\sqrt{\eta}$. 
Finally, when $\vartheta \geq \vartheta_{4412}$ as given by equation (A.14), also the $\operatorname{arc} C_{44}$ lies completely in the interior of $\mathcal{R}_{\oplus}$ and we need the intersection between $C_{12}$ and $C_{14}$ (Fig. $7 b$ ). On $C_{12}, t \in\left[0, t_{1214}\right]$ and on $C_{14}, t \in\left[t_{1412}, 2 \alpha\right]$ (see equations (A.15) and (A.16)).

In this case the excluded area is given by

$$
\begin{aligned}
A(\alpha, \vartheta, \eta)= & 2\left[\frac{1}{2} \operatorname{Sett}(2 \eta, \vartheta-2 \alpha)+\operatorname{Sett}\left(1+2 \eta, 2 \alpha-t_{1412}\right)+\operatorname{Sett}\left(1+2 \eta, t_{1214}\right)+\frac{1}{2} \operatorname{Sett}(2 \eta, 2 \pi-2 \alpha-\vartheta)\right. \\
& \left.+\mathcal{T}\left(h_{1}^{\prime \prime}, b_{1}^{\prime}, b_{2}^{\prime}\right)+\mathcal{T}\left(h_{2}^{\prime \prime}, b_{2}^{\prime}, b_{5}\right)\right]
\end{aligned}
$$

where

$$
b_{2}^{\prime}=x_{14}\left(t_{1412}\right) \quad h_{1}^{\prime \prime}=y_{14}(2 \alpha)-y_{14}\left(t_{1412}\right) \quad h_{2}^{\prime \prime}=y_{12}\left(t_{1214}\right)-y_{12}(0) .
$$

When values $\eta \ll 1$ are considered, then

$$
A(\alpha, \vartheta, \eta)=4 \alpha+2 \sin 2 \alpha \cos \vartheta+16 \alpha \eta+O\left(\eta^{2}\right) .
$$

Finally, by collecting together equations (III.16), (III.19), (III.22), and (III.28) and neglecting powers in $\eta$ higher than one, we obtain a simple approximation $A_{0}$ for the excluded area:

$$
A_{0}(\eta, \alpha, \theta):= \begin{cases}2 \alpha-\vartheta+2 \sin \vartheta-\sin (2 \alpha+\vartheta)+16 \alpha \eta & \vartheta \in[0,2 \alpha] \\ 4 \sin \vartheta \sin ^{2} \alpha+16 \alpha \eta & \vartheta \in[2 \alpha, \pi-2 \alpha] \\ 2(\vartheta+2 \alpha-\pi)+2 \sin \vartheta+2 \cos \vartheta \sin 2 \alpha+16 \alpha \eta & \vartheta \in[\pi-2 \alpha, \vartheta a \\ 4 \alpha+2 \sin 2 \alpha \cos \vartheta+16 \alpha \eta & \vartheta \in\left[\vartheta_{a}(\alpha, \eta), \pi\right]\end{cases}
$$

\section{B. Boomerangs II: $\alpha \in\left[\frac{\pi}{4}, \frac{\pi}{2}\right]$}

When $\alpha \in\left[\frac{\pi}{4}, \frac{\pi}{2}\right]$ and so $\pi-2 \alpha<2 \alpha$, it can be checked that, when $\vartheta \in[0, \pi-2 \alpha]$, the reduced boundary $\mathcal{R}_{\oplus}$ coincides with that obtained in Subsection III A 1. Moreover, $\vartheta_{4312}>2 \alpha$ and the results for $\vartheta \in[2 \alpha, \pi]$ are the same as in Subsections III A 3-III A 5. New computations are needed only when $\vartheta \in[\pi-2 \alpha, 2 \alpha]$ where the reduced boundary $\mathcal{R}_{\oplus}$ is formed by the arcs

\begin{tabular}{|l|l|l|l|l|}
\hline arc & radius & center & $t$ & base curve \\
\hline$C_{11}$ & $2(1+\eta)$ & $O$ & {$\left[\alpha-\frac{\vartheta}{2}, 2 \alpha-\vartheta\right]$} & $\mathcal{K}_{1}(t)$ \\
$C_{14}$ & $1+2 \eta$ & $B^{\prime}$ & {$[0, \vartheta]$} & $\mathcal{C}_{1}(t)$ \\
$C_{44}$ & $2 \eta$ & $B+B^{\prime}$ & {$[0, \pi-\vartheta]$} & $\mathcal{K}_{4}(t)$ \\
$C_{43}$ & $2 \eta-1$ & $B$ & {$\left[\vartheta, t_{4312}\right]$} & $\mathcal{C}_{4}(t)$ \\
$C_{12}$ & $1+2 \eta$ & $A^{\prime}$ & {$\left[0, t_{1243}\right]$} & $\mathcal{C}_{1}(t)$ \\
$C_{42}$ & $2 \eta$ & $B+A^{\prime}$ & {$\left[(\pi-\alpha)-\frac{\vartheta}{2}, 2(\pi-\alpha)-\vartheta\right]$} & $\mathcal{K}_{2}(t)$ \\
\hline
\end{tabular}

where $t_{1243}$ and $t_{4312}$ are given by equations (A.5) and (A.6). The excluded area is then given by

$$
\begin{aligned}
A(\alpha, \vartheta, \eta)= & 2\left[\frac{1}{2} \operatorname{Sett}(2(1+\eta), 2 \alpha-\vartheta)+\operatorname{Sett}(1+2 \eta, \vartheta)+\operatorname{Sett}(2 \eta, \pi-\vartheta)-\operatorname{Sett}\left(2 \eta-1, t_{4312}-\vartheta\right)\right. \\
& +\operatorname{Sett}\left(1+2 \eta, t_{1243}\right)+\frac{1}{2} \operatorname{Sett}(2 \eta, 2(\pi-\alpha)-\vartheta)+\mathcal{T}\left(h_{1}, b_{1}, b_{2}\right)+\mathcal{T}\left(h_{2}, b_{2}, b_{3}\right) \\
& \left.+\mathcal{T}\left(h_{3}^{\prime}, b_{3}, b_{4}^{\prime}\right)+\mathcal{T}\left(h_{4}, b_{4}^{\prime}, b_{5}\right)\right]
\end{aligned}
$$

where the bases and the heights of the trapezia are given in equations (III.14), (III.15), and (III.21). In the limit when $\eta \ll 1$ it is possible to check that

$$
A(\alpha, \vartheta, \eta)=6 \alpha-2 \pi+\vartheta+2 \sin \vartheta+\sin (\vartheta+2 \alpha)+16 \alpha \eta+O\left(\eta^{2}\right) .
$$

The excluded area within this approximation is then

$$
A_{0}(\eta, \alpha, \theta):= \begin{cases}2 \alpha-\vartheta+2 \sin \vartheta-\sin (2 \alpha+\vartheta)+16 \alpha \eta & \vartheta \in[0, \pi-2 \alpha] \\ 6 \alpha-2 \pi+\vartheta+2 \sin \vartheta+\sin (\vartheta+2 \alpha)+16 \alpha \eta & \vartheta \in[\pi-2 \alpha, 2 \alpha] \\ 2(\vartheta+2 \alpha-\pi)+2 \sin \vartheta+2 \cos \vartheta \sin 2 \alpha+16 \alpha \eta & \vartheta \in\left[2 \alpha, \vartheta_{a}(\alpha, \eta)\right] \\ 4 \alpha+2 \sin 2 \alpha \cos \vartheta+16 \alpha \eta & \vartheta \in\left[\vartheta_{a}(\alpha, \eta), \pi\right]:\end{cases}
$$

as remarked before, this approximation does not hold if $\alpha$ is too close to $\frac{\pi}{2}$. 


\section{Horseshoes I: $\alpha \in\left[\frac{\pi}{2}, \alpha^{\mathrm{cr}}(\eta)\right]$}

When $\alpha$ exceeds $\pi / 2$, the trimming procedure to obtain the boundary of the Minkowski sum $\mathcal{M} \oplus \mathcal{M}^{\prime}$ from the convolution curve $\partial \mathcal{M} * \partial \mathcal{M}^{\prime}$ becomes more delicate. It is crucial to determine the magnitude of $\vartheta_{4312}$ and $\vartheta_{4412}$ as compared to $2 \alpha-\pi$ and $2(\pi-\alpha)$. To this aim, we impose that $\vartheta=2 \alpha-\pi$ or $\vartheta=2(\pi-\alpha)$ solve equations (A.7) or (A.13), respectively. It can be checked that both $\vartheta_{4312}$ and $\vartheta_{4412}$ are less than $2(\pi-\alpha)$, for any value of $\alpha$ and $\eta$. On the other hand, $\vartheta_{4312}>2 \alpha-\pi$ if and only if

$$
\alpha<\alpha_{\mathrm{cr}}(\eta):=\frac{\pi}{2}+\frac{1}{2} \arccos (2 \eta) \in\left[\frac{\pi}{2}, \frac{3 \pi}{4}\right]
$$

whereas $\vartheta_{4412}>2 \alpha-\pi$ if and only if

$$
\alpha<\alpha_{\mathrm{cr}}^{*}(\eta):=\frac{\pi}{2}+\frac{1}{2} \arccos \left(\frac{\eta}{1+2 \eta}\right) \in\left[\frac{\pi}{2}, \frac{3 \pi}{4}\right],
$$

with $\alpha_{\mathrm{cr}}<\alpha_{\mathrm{cr}}^{*}$. A new critical value of $\vartheta$ replacing $\vartheta=\vartheta_{4312}$ has to be considered. In fact, when $\alpha>\pi / 2$ it is possible (see Figure 12) that $C_{12}$ becomes tangent to $C_{44}$ when $\vartheta=\vartheta_{1244}^{*}(\alpha, \eta)<\vartheta_{4312}$ : when this happens, the excluded area suffers a tiny jump, as a function of $\vartheta$.

The angle $\vartheta_{1244}^{*}$ is defined as the value of $\vartheta$ at which the radicand in equation (A.12) vanishes, that is,

$$
\vartheta=\vartheta_{1244}^{*}(\alpha, \eta):=2 \arctan \left(\frac{\nu(\alpha)+\sqrt{\nu(\alpha)^{2}+\mu(\alpha)^{2}-\omega(\eta, \alpha)^{2}}}{\mu(\alpha)+\omega(\eta, \alpha)}\right)
$$

where we set

$$
\mu(\alpha):=\sin ^{2} \alpha \quad \nu(\alpha):=\sin \alpha \cos \alpha \quad \omega(\eta, \alpha):=2 \eta(2 \eta+1)-\sin ^{2} \alpha .
$$

However, the tangency between $C_{12}$ and $C_{44}$ affects the structure of the convolution curve if (see equation (A.12)) $t_{4412}:=t\left(\vartheta_{1244}^{*}\right)$ lies in the interval $[0, \pi-\vartheta]$ where $C_{44}$ belongs to $\mathcal{R}_{\oplus}$. As can be seen from Fig. $13, \vartheta_{1244}^{*} \leq \vartheta_{4312}$ and the difference between the two functions is not easy to perceive for most values of $\alpha$.

$$
\text { 1. } \vartheta \in\left[0, \vartheta_{1244}^{*}\right]
$$

The arcs that contribute to the excluded area are:

\begin{tabular}{|l|l|l|l|l|}
\hline arc & radius & center & $t$ & base curve \\
\hline$C_{11}$ & $2(\eta+1)$ & $O$ & {$\left[0, \alpha-\frac{\vartheta}{2}\right]$} & $\mathcal{K}_{1}(t)$ \\
$C_{14}$ & $1+2 \eta$ & $B^{\prime}$ & {$[0, \vartheta]$} & $\mathcal{C}_{1}(t)$ \\
$C_{44}$ & $2 \eta$ & $B+B^{\prime}$ & {$[0, \pi-\vartheta]$} & $\mathcal{K}_{4}(t)$ \\
$C_{43}$ & $2 \eta-1$ & $B$ & {$\left[\vartheta, t_{4312}\right]$} & $\mathcal{C}_{4}(t)$ \\
$C_{12}$ & $1+2 \eta$ & $A^{\prime}$ & {$\left[0, t_{1243}\right]$} & $\mathcal{C}_{1}(t)$ \\
$C_{42}$ & $2 \eta$ & $B+A^{\prime}$ & {$\left[\pi-\alpha-\frac{\vartheta}{2}, 2(\pi-\alpha)-\vartheta\right]$} & $\mathcal{K}_{2}(t)$ \\
\hline
\end{tabular}

The excluded area is then given by

$$
\begin{aligned}
A(\alpha, \vartheta, \eta)= & 2\left[\frac{1}{2} \operatorname{Sett}(2(1+\eta), 2 \alpha-\vartheta)+\operatorname{Sett}(1+2 \eta, \vartheta)+\operatorname{Sett}(2 \eta, \pi-\vartheta)-\operatorname{Sett}\left(2 \eta-1, t_{4312}-\vartheta\right)\right. \\
& +\operatorname{Sett}\left(1+2 \eta, t_{1243}\right)+\frac{1}{2} \operatorname{Sett}(2 \eta, 2(\pi-\alpha)-\vartheta)+\mathcal{T}\left(h_{1}, b_{1}, b_{2}\right)+\mathcal{T}\left(h_{2}, b_{2}, b_{3}\right)+\mathcal{T}\left(h_{3}^{\prime}, b_{3}, b_{4}^{\prime}\right) \\
& \left.+\mathcal{T}\left(h_{4}, b_{4}^{\prime}, b_{5}\right)\right]
\end{aligned}
$$

where the bases and the heights of the trapezia are given by equations (III.14), (III.15), and (III.21). We note that the heights $h_{2}$ and $h_{3}^{\prime}$ could be negative, as it should be to obtain the correct value of the excluded area. 


$$
\text { 2. } \vartheta \in\left[\vartheta_{1244}^{*}, \vartheta_{4412}\right]
$$

When $C_{12}$ and $C_{44}$ touch at a point on the set $\mathcal{R}_{\oplus}$ we need to consider values $t \in\left[0, t_{1244}\right]$ along $C_{12}$ and values $t \in\left[\vartheta, t_{4412}\right]$ along $C_{44}$, while $C_{43}$ is trapped in the interior of $\mathcal{R}_{\oplus}$. In this regime, the following arcs contribute to the reduced boundary $\mathcal{R}_{\oplus}$ :

\begin{tabular}{|l|l|l|l|l|}
\hline arc & radius & center & $t$ & base curve \\
\hline$C_{11}$ & $2(1+\eta)$ & $O$ & {$\left[0, \alpha-\frac{\vartheta}{2}\right]$} & $\mathcal{K}_{1}(t)$ \\
$C_{14}$ & $1+2 \eta$ & $B^{\prime}$ & {$[0, \vartheta]$} & $\mathcal{C}_{1}(t)$ \\
$C_{44}$ & $2 \eta$ & $B+B^{\prime}$ & {$\left[t_{4412}, \pi-\vartheta\right]$} & $\mathcal{K}_{4}(t)$ \\
$C_{12}$ & $1+2 \eta$ & $A^{\prime}$ & {$\left[0, t_{1244}\right]$} & $\mathcal{C}_{1}(t)$ \\
$C_{42}$ & $2 \eta$ & $B+A^{\prime}$ & {$\left[(\pi-\alpha)-\frac{\vartheta}{2}, 2(\pi-\alpha)-\vartheta\right]$} & $\mathcal{K}_{2}(t)$ \\
\hline
\end{tabular}

where $t_{1214}$ and $t_{1412}$ are given in equations (A.15) and (A.16). The excluded area is given by

$$
\begin{aligned}
A(\alpha, \vartheta, \eta)= & 2\left[\frac{1}{2} \operatorname{Sett}(2(1+\eta), 2 \alpha-\vartheta)+\operatorname{Sett}(1+2 \eta, \vartheta)+\operatorname{Sett}\left(2 \eta, \pi-\vartheta-t_{4412}\right)+\operatorname{Sett}\left(1+2 \eta, t_{1244}\right)\right. \\
& \left.+\frac{1}{2} \operatorname{Sett}(2 \eta, 2(\pi-\alpha)-\vartheta)+\mathcal{T}\left(h_{1}, b_{1}, b_{2}\right)+\mathcal{T}\left(h_{2}^{\prime}, b_{2}, b_{3}^{\prime}\right)+\mathcal{T}\left(h_{3}^{\prime \prime}, b_{3}^{\prime}, b_{5}\right)\right]
\end{aligned}
$$

where equations (III.14), (III.15), (III.21), and (III.24) have been employed too.

$$
\text { 3. } \vartheta \in\left[\vartheta_{4412}, 2(\pi-\alpha)\right]
$$

The arc $C_{44}$ no longer contributes to the set $\partial\left(\mathcal{M} \oplus \mathcal{M}^{\prime}\right)$ and the $\operatorname{arcs} C_{12}$ and $C_{14}$ intersect each other. The reduced boundary $\mathcal{R}_{\oplus}$ is formed by the following arcs

\begin{tabular}{|l|l|l|l|l|}
\hline arc & radius & center & $t$ & base curve \\
\hline$C_{11}$ & $2(1+\eta)$ & $O$ & {$\left[0, \alpha-\frac{\vartheta}{2}\right]$} & $\mathcal{K}_{1}(t)$ \\
$C_{14}$ & $1+2 \eta$ & $B^{\prime}$ & {$\left[t_{1412}, \vartheta\right]$} & $\mathcal{C}_{1}(t)$ \\
$C_{12}$ & $1+2 \eta$ & $A^{\prime}$ & {$\left[0, t_{1214}\right]$} & $\mathcal{C}_{1}(t)$ \\
$C_{42}$ & $2 \eta$ & $B+A^{\prime}$ & {$\left[(\pi-\alpha)-\frac{\vartheta}{2}, 2(\pi-\alpha)-\vartheta\right]$} & $\mathcal{K}_{2}(t)$ \\
\hline
\end{tabular}

Correspondingly, the excluded area is given by

$$
\begin{aligned}
A(\alpha, \vartheta, \eta)= & 2\left[\frac{1}{2} \operatorname{Sett}(2(1+\eta), 2 \alpha-\vartheta)+\operatorname{Sett}\left(1+2 \eta, \vartheta-t_{1412}\right)+\operatorname{Sett}\left(1+2 \eta, t_{1214}\right)\right. \\
& \left.+\frac{1}{2} \operatorname{Sett}(2 \eta, 2(\pi-\alpha)-\vartheta)+\mathcal{T}\left(h_{1}^{\prime \prime \prime}, b_{1}, b_{2}^{\prime}\right)+\mathcal{T}\left(h_{2}^{\prime \prime}, b_{2}^{\prime}, b_{5}\right)\right]
\end{aligned}
$$

where use of equations (III.14), (III.21) and (III.27) has been made together with

$$
\begin{gathered}
h_{1}^{\prime \prime \prime}=\left[y_{14}(\vartheta)-y_{14}^{\mathrm{bis}}\left(t_{1412}\right)\right] . \\
\text { 4. } \quad \vartheta \in[2(\pi-\alpha), \pi]
\end{gathered}
$$

Here the arc $C_{42}$ is replaced by the arc $C_{11}^{\text {bis }}$ with scaled radius $2(1+\eta)$, centered at $O$, based on the curve $\mathcal{C}_{1}$ and symmetric about the $y$-axis. On $\mathcal{R}_{\oplus}, t \in\left[\frac{\vartheta}{2}-(\pi-\alpha), \vartheta-2(\pi-\alpha)\right]$ along $C_{11}^{\text {bis }}$. Moreover, the $\operatorname{arc} C_{12}$ is replaced by $C_{12}^{\text {bis }}$ that differs from $C_{12}$ only for the base curve and along which $t \in\left[0, t_{1214}^{\text {bis }}\right]$. The reduced boundary is formed by the following arcs:

\begin{tabular}{|l|l|l|l|l|}
\hline arc & radius & center & $t$ & base curve \\
\hline$C_{11}$ & $2(1+\eta)$ & $O$ & {$\left[0, \alpha-\frac{\vartheta}{2}\right]$} & $\mathcal{K}_{1}(t)$ \\
$C_{14}$ & $1+2 \eta$ & $B^{\prime}$ & {$\left[t_{1412}, \vartheta\right]$} & $\mathcal{C}_{1}(t)$ \\
$C_{12}^{\text {bis }}$ & $1+2 \eta$ & $A^{\prime}$ & {$\left[0, t_{1214}^{\text {bis }}\right]$} & $\mathcal{K}_{2}(t)$ \\
$C_{11}^{\text {bis }}$ & $2(1+\eta)$ & $O$ & {$\left[\frac{\vartheta}{2}-(\pi-\alpha), \vartheta-2(\pi-\alpha)\right]$} & $\mathcal{C}_{1}(t)$ \\
\hline
\end{tabular}


The value of $t_{1412}$ is still given by (A.16), while the value $t_{1214}^{\text {bis }}$ can be obtained in the same way as $t_{1214}($ see equation (A.15)) apart from the different parametrization of the arc $C_{12}^{\text {bis }}$ as compared to $C_{12}$. As a result, $t_{1214}^{\text {bis }}$ attains the value

$$
t_{1214}^{\mathrm{bis}}:=\pi-\alpha+\arcsin \left(\frac{\sin \alpha}{1+2 \eta}\right) .
$$

Then, the excluded area is now given by

$$
\begin{aligned}
A(\alpha, \vartheta, \eta)= & 2\left[\frac{1}{2} \operatorname{Sett}(2(1+\eta), 2 \alpha-\vartheta)+\operatorname{Sett}\left(1+2 \eta, \vartheta-t_{1412}\right)+\operatorname{Sett}\left(1+2 \eta, t_{1214}^{\mathrm{bis}}\right)\right. \\
& \left.+\frac{1}{2} \operatorname{Sett}(2(1+\eta), \vartheta-2(\pi-\alpha))+\mathcal{T}\left(h_{1}^{\prime \prime \prime}, b_{1}, b_{2}^{\prime}\right)+\mathcal{T}\left(h_{2}^{\prime \prime \prime}, b_{2}^{\prime}, b_{3}^{\prime \prime}\right)\right]
\end{aligned}
$$

where

$$
b_{3}^{\prime \prime}=x_{12}^{\mathrm{bis}}(0) \quad h_{2}^{\prime \prime \prime}=y_{12}^{\mathrm{bis}}\left(t_{1214}\right)-y_{12}^{\mathrm{bis}}(0)
$$

\section{Horseshoes II: $\alpha \in\left[\alpha^{\mathrm{cr}}, \alpha^{\mathrm{cr} *}\right]$}

Here we need to distinguish the following regimes, according to whether $\vartheta \in\left[0, \vartheta_{1244}^{*}\right], \vartheta \in\left[\vartheta_{1244}^{*}, 2 \alpha-\pi\right], \vartheta \in$ $\left[2 \alpha-\pi, \vartheta_{4412}\right], \vartheta \in\left[\vartheta_{4412}, 2(\pi-\alpha)\right]$ or $\vartheta \in[2(\pi-\alpha), \pi]$.

$$
\text { 1. } \vartheta \in\left[0, \vartheta_{1244}^{*}\right]
$$

The reduced boundary $\mathcal{R}_{\oplus}$ is formed by the arcs

\begin{tabular}{|l|l|l|l|l|}
\hline arc & radius & center & $t$ & base curve \\
\hline$C_{11}$ & $2(1+\eta)$ & $O$ & {$\left[0, \alpha-\frac{\vartheta}{2}\right]$} & $\mathcal{K}_{1}(t)$ \\
$C_{14}$ & $1+2 \eta$ & $B^{\prime}$ & {$[0, \vartheta]$} & $\mathcal{C}_{1}(t)$ \\
$C_{44}$ & $2 \eta$ & $B+B^{\prime}$ & {$[0, \pi-\vartheta]$} & $\mathcal{K}_{4}(t)$ \\
$C_{43}$ & $2 \eta-1$ & $B$ & {$\left[\vartheta, t_{4312}\right]$} & $\mathcal{C}_{4}(t)$ \\
$C_{12}$ & $1+2 \eta$ & $A^{\prime}$ & {$\left[0, t_{1243}\right]$} & $\mathcal{C}_{1}(t)$ \\
$C_{42}$ & $2 \eta$ & $B+A^{\prime}$ & {$\left[\pi-\alpha-\frac{\vartheta}{2}, 2(\pi-\alpha)-\vartheta\right]$} & $\mathcal{K}_{2}(t)$ \\
\hline
\end{tabular}

and the excluded area is given by

$$
\begin{aligned}
A(\alpha, \vartheta, \eta)= & 2\left[\frac{1}{2} \operatorname{Sett}(2(1+\eta), 2 \alpha-\vartheta)+\operatorname{Sett}(1+2 \eta, \vartheta)+\operatorname{Sett}(2 \eta, \pi-\vartheta)-\operatorname{Sett}\left(2 \eta-1, t_{4312}-\vartheta\right)\right. \\
& +\operatorname{Sett}\left(1+2 \eta, t_{1243}\right)+\frac{1}{2} \operatorname{Sett}(2 \eta, 2(\pi-\alpha)-\vartheta)+\mathcal{T}\left(h_{1}, b_{1}, b_{2}\right) \\
& \left.+\mathcal{T}\left(h_{2}, b_{2}, b_{3}\right)+\mathcal{T}\left(h_{3}^{\prime}, b_{3}, b_{4}^{\prime}\right)+\mathcal{T}\left(h_{4}, b_{4}^{\prime}, b_{5}\right)\right]
\end{aligned}
$$$$
\text { 2. } \vartheta \in\left[\vartheta_{4412}^{*}, \vartheta_{4412}\right]
$$

The reduced boundary is formed by the arcs

\begin{tabular}{|l|l|l|l|l|}
\hline arc & radius & center & $t$ & base curve \\
\hline$C_{11}$ & $2(1+\eta)$ & $O$ & {$\left[0, \alpha-\frac{\vartheta}{2}\right]$} & $\mathcal{K}_{1}(t)$ \\
$C_{14}$ & $1+2 \eta$ & $B^{\prime}$ & {$[0, \vartheta]$} & $\mathcal{C}_{1}(t)$ \\
$C_{44}$ & $2 \eta$ & $B+B^{\prime}$ & {$\left[t_{4412}, \pi-\vartheta\right]$} & $\mathcal{K}_{4}(t)$ \\
$C_{12}$ & $1+2 \eta$ & $A^{\prime}$ & {$\left[0, t_{1244}\right]$} & $\mathcal{C}_{1}(t)$ \\
$C_{42}$ & $2 \eta$ & $B+A^{\prime}$ & {$\left[(\pi-\alpha)-\frac{\vartheta}{2}, 2(\pi-\alpha)-\vartheta\right]$} & $\mathcal{K}_{2}(t)$ \\
\hline
\end{tabular}

and the excluded area is

$$
\begin{aligned}
A(\alpha, \vartheta, \eta)= & 2\left[\frac{1}{2} \operatorname{Sett}(2(1+\eta), 2 \alpha-\vartheta)+\operatorname{Sett}(1+2 \eta, \vartheta)+\operatorname{Sett}\left(2 \eta, \pi-\vartheta-t_{4412}\right)+\operatorname{Sett}\left(1+2 \eta, t_{1244}\right)\right. \\
& \left.+\frac{1}{2} \operatorname{Sett}(2 \eta, 2(\pi-\alpha)-\vartheta)+\mathcal{T}\left(h_{1}, b_{1}, b_{2}\right)+\mathcal{T}\left(h_{2}^{\prime}, b_{2}, b_{3}^{\prime}\right)+\mathcal{T}\left(h_{3}^{\prime \prime}, b_{3}^{\prime}, b_{5}\right)\right]
\end{aligned}
$$


3. $\vartheta \in\left[\vartheta_{4412}, 2(\pi-\alpha)\right]$

The essential boundary is formed by the arcs

\begin{tabular}{|l|l|l|l|l|}
\hline arc & radius & center & $t$ & base curve \\
\hline$C_{11}$ & $2(1+\eta)$ & $O$ & {$\left[0, \alpha-\frac{\vartheta}{2}\right]$} & $\mathcal{K}_{1}(t)$ \\
$C_{14}$ & $1+2 \eta$ & $B^{\prime}$ & {$\left[t_{1412}, \vartheta\right]$} & $\mathcal{C}_{1}(t)$ \\
$C_{12}$ & $1+2 \eta$ & $A^{\prime}$ & {$\left[0, t_{1214}\right]$} & $\mathcal{C}_{1}(t)$ \\
$C_{42}$ & $2 \eta$ & $B+A^{\prime}$ & {$\left[\pi-\alpha-\frac{\vartheta}{2}, 2(\pi-\alpha)-\vartheta\right]$} & $\mathcal{K}_{2}(t)$ \\
\hline
\end{tabular}

and the excluded area is

$$
\begin{gathered}
A(\alpha, \vartheta, \eta)=2\left[\frac{1}{2} \operatorname{Sett}(2(1+\eta), 2 \alpha-\vartheta)+\operatorname{Sett}\left(1+2 \eta, \vartheta-t_{1412}\right)+\operatorname{Sett}\left(1+2 \eta, t_{1214}\right)\right. \\
\left.+\frac{1}{2} \operatorname{Sett}(2 \eta, 2(\pi-\alpha)-\vartheta)+\mathcal{T}\left(h_{1}^{\prime \prime \prime}, b_{1}, b_{2}^{\prime}\right)+\mathcal{T}\left(h_{2}^{\prime \prime \prime}, b_{2}^{\prime}, b_{5}\right)\right] . \\
\text { 4. } \vartheta \in[2(\pi-\alpha), \pi]
\end{gathered}
$$

When $\vartheta$ ranges in this interval, the excluded region coincides with that found in Section (III C 4) for $\vartheta$ ranging in the same interval, and the excluded area is given by equation (III.41).

\section{E. Horseshoes III: $\alpha \in\left[\alpha^{\mathrm{cr} *}, \alpha_{0}\right]$}

The lower bound of this interval is the value $\alpha^{\mathrm{cr} *}$ of $\alpha$ at which $\vartheta_{4412}=2 \alpha-\pi$ and the upper bound is the value $\alpha_{0}$ of $\alpha$ at which $\vartheta_{1244}^{*}=0$. By use of equation (III.35) we obtain

$$
\alpha_{0}:=\pi-\arcsin (\sqrt{\eta(2 \eta+1)}) .
$$

1. $\vartheta \in\left[0, \vartheta_{1244}^{*}\right]$

The reduced boundary is formed by the arcs

\begin{tabular}{|l|l|l|l|l|}
\hline arc & radius & center & $t$ & base curve \\
\hline$C_{11}$ & $2(1+\eta)$ & $O$ & {$\left[0, \alpha-\frac{\vartheta}{2}\right]$} & $\mathcal{K}_{1}(t)$ \\
$C_{14}$ & $1+2 \eta$ & $B^{\prime}$ & {$[0, \vartheta]$} & $\mathcal{C}_{1}(t)$ \\
$C_{44}$ & $2 \eta$ & $B+B^{\prime}$ & {$[0, \pi-\vartheta]$} & $\mathcal{K}_{4}(t)$ \\
$C_{43}$ & $2 \eta-1$ & $B$ & {$\left[\vartheta, t_{4312}\right]$} & $\mathcal{C}_{4}(t)$ \\
$C_{12}$ & $1+2 \eta$ & $A^{\prime}$ & {$\left[0, t_{1243}\right]$} & $\mathcal{C}_{1}(t)$ \\
$C_{42}$ & $2 \eta$ & $B+A^{\prime}$ & {$\left[\pi-\alpha-\frac{\vartheta}{2}, 2(\pi-\alpha)-\vartheta\right]$} & $\mathcal{K}_{2}(t)$ \\
\hline
\end{tabular}

and the excluded area is given by

$$
\begin{aligned}
A(\alpha, \vartheta, \eta)= & 2\left[\frac{1}{2} \operatorname{Sett}(2(1+\eta), 2 \alpha-\vartheta)+\operatorname{Sett}(1+2 \eta, \vartheta)+\operatorname{Sett}(2 \eta, \pi-\vartheta)-\operatorname{Sett}\left(2 \eta-1, t_{4312}-\vartheta\right)\right. \\
& +\operatorname{Sett}\left(1+2 \eta, t_{1243}\right)+\frac{1}{2} \operatorname{Sett}(2 \eta, 2(\pi-\alpha)-\vartheta)+\mathcal{T}\left(h_{1}, b_{1}, b_{2}\right) \\
& \left.+\mathcal{T}\left(h_{2}, b_{2}, b_{3}\right)+\mathcal{T}\left(h_{3}^{\prime}, b_{3}, b_{4}^{\prime}\right)+\mathcal{T}\left(h_{4}, b_{4}^{\prime}, b_{5}\right)\right]
\end{aligned}
$$

$$
\text { 2. } \vartheta \in\left[\vartheta_{1244}^{*}, \vartheta_{4412}\right]
$$

The essential boundary is formed by the arcs 


\begin{tabular}{|l|l|l|l|l|}
\hline arc & radius & center & $t$ & base curve \\
\hline$C_{11}$ & $2(1+\eta)$ & $O$ & {$\left[0, \alpha-\frac{\vartheta}{2}\right]$} & $\mathcal{K}_{1}(t)$ \\
$C_{14}$ & $1+2 \eta$ & $B^{\prime}$ & {$[0, \vartheta]$} & $\mathcal{C}_{1}(t)$ \\
$C_{44}$ & $2 \eta$ & $B+B^{\prime}$ & {$\left[t_{4412}, \pi-\vartheta\right]$} & $\mathcal{K}_{4}(t)$ \\
$C_{12}$ & $1+2 \eta$ & $A^{\prime}$ & {$\left[0, t_{1244}\right]$} & $\mathcal{C}_{1}(t)$ \\
$C_{42}$ & $2 \eta$ & $B+A^{\prime}$ & {$\left[\pi-\alpha-\frac{\vartheta}{2}, 2(\pi-\alpha)-\vartheta\right]$} & $\mathcal{K}_{2}(t)$ \\
\hline
\end{tabular}

and the excluded area is

$$
\begin{aligned}
& A(\alpha, \vartheta, \eta)=2\left[\frac{1}{2} \operatorname{Sett}(2(1+\eta), 2 \alpha-\vartheta)+\operatorname{Sett}(1+2 \eta, \vartheta)+\operatorname{Sett}\left(2 \eta, \pi-\vartheta-t_{4412}\right)+\operatorname{Sett}\left(1+2 \eta, t_{1244}\right)\right. \\
& \left.+\frac{1}{2} \operatorname{Sett}(2 \eta, 2(\pi-\alpha)-\vartheta)+\mathcal{T}\left(h_{1}, b_{1}, b_{2}\right)+\mathcal{T}\left(h_{2}^{\prime}, b_{2}, b_{3}^{\prime}\right)+\mathcal{T}\left(h_{3}^{\prime \prime}, b_{3}^{\prime}, b_{5}\right)\right] . \\
& \text { 3. } \vartheta \in\left[\vartheta_{4412}, 2(\pi-\alpha)\right]
\end{aligned}
$$

The essential boundary is formed by the arcs

\begin{tabular}{|l|l|l|l|l|}
\hline arc & radius & center & $t$ & base curve \\
\hline$C_{11}$ & $2(1+\eta)$ & $O$ & {$\left[0, \alpha-\frac{\vartheta}{2}\right]$} & $\mathcal{K}_{1}(t)$ \\
$C_{14}$ & $1+2 \eta$ & $B^{\prime}$ & {$\left[t_{1412}, \vartheta\right]$} & $\mathcal{C}_{1}(t)$ \\
$C_{12}$ & $1+2 \eta$ & $A^{\prime}$ & {$\left[0, t_{1214}\right]$} & $\mathcal{C}_{1}(t)$ \\
$C_{42}$ & $2 \eta$ & $B+A^{\prime}$ & {$\left[\pi-\alpha-\frac{\vartheta}{2}, 2(\pi-\alpha)-\vartheta\right]$} & $\mathcal{K}_{2}(t)$ \\
\hline
\end{tabular}

and the excluded area is

$$
\begin{aligned}
& A(\alpha, \vartheta, \eta)=2\left[\frac{1}{2} \operatorname{Sett}(2(1+\eta), 2 \alpha-\vartheta)+\operatorname{Sett}\left(1+2 \eta, \vartheta-t_{1412}\right)+\operatorname{Sett}\left(1+2 \eta, t_{1214}\right)\right. \\
& \left.+\frac{1}{2} \operatorname{Sett}(2 \eta, 2(\pi-\alpha)-\vartheta)+\mathcal{T}\left(h_{1}^{\prime \prime \prime}, b_{1}, b_{2}^{\prime}\right)+\mathcal{T}\left(h_{2}^{\prime \prime}, b_{2}^{\prime}, b_{5}\right)\right] \text {. } \\
& \text { 4. } \vartheta \in[2(\pi-\alpha), \pi]
\end{aligned}
$$

The essential boundary is formed by the arcs

\begin{tabular}{|l|l|l|l|l|}
\hline arc & radius & center & $t$ & base curve \\
\hline$C_{11}$ & $2(1+\eta)$ & $O$ & {$\left[0, \alpha-\frac{\vartheta}{2}\right]$} & $\mathcal{K}_{1}(t)$ \\
$C_{14}$ & $1+2 \eta$ & $B^{\prime}$ & {$\left[t_{1412}, \vartheta\right]$} & $\mathcal{C}_{1}(t)$ \\
$C_{12}^{\text {bis }}$ & $1+2 \eta$ & $A^{\prime}$ & {$\left[0, t_{1214}^{\text {bis }}\right]$} & $\mathcal{K}_{2}(t)$ \\
$C_{11}^{\text {bis }}$ & $2(1+\eta)$ & $O$ & {$\left[\frac{\vartheta}{2}-\pi+\alpha, \vartheta-2(\pi-\alpha)\right]$} & $\mathcal{C}_{1}(t)$ \\
\hline
\end{tabular}

and the excluded area is

$$
\begin{aligned}
A(\alpha, \vartheta, \eta)= & 2\left[\frac{1}{2} \operatorname{Sett}(2(1+\eta), 2 \alpha-\vartheta)+\operatorname{Sett}\left(1+2 \eta, \vartheta-t_{1412}\right)+\operatorname{Sett}\left(1+2 \eta, t_{1214}^{\mathrm{bis}}\right)\right. \\
& \left.+\frac{1}{2} \operatorname{Sett}(2(1+\eta), \vartheta-2(\pi-\alpha))+\mathcal{T}\left(h_{1}^{\prime \prime \prime}, b_{1}, b_{2}^{\prime}\right)+\mathcal{T}\left(h_{2}^{\prime \prime \prime}, b_{2}^{\prime}, b_{3}^{\prime \prime}\right)\right] .
\end{aligned}
$$

\section{F. Horseshoes IV: $\alpha \in\left[\alpha_{0}, \alpha_{m}\right]$}

For these values of $\alpha, \vartheta_{1244}^{*}<0$ and so the arc $C_{43}$ never enters the reduced boundary. As a result, only three regimes survive. 
The essential boundary is formed by the arcs

\begin{tabular}{|l|l|l|l|l|}
\hline arc & radius & center & $t$ & base curve \\
\hline$C_{11}$ & $2(1+\eta)$ & $O$ & {$\left[0, \alpha-\frac{\vartheta}{2}\right]$} & $\mathcal{K}_{1}(t)$ \\
$C_{14}$ & $1+2 \eta$ & $B^{\prime}$ & {$[0, \vartheta]$} & $\mathcal{C}_{1}(t)$ \\
$C_{44}$ & $2 \eta$ & $B+B^{\prime}$ & {$\left[t_{4412}, \pi-\vartheta\right]$} & $\mathcal{K}_{4}(t)$ \\
$C_{12}$ & $1+2 \eta$ & $A^{\prime}$ & {$\left[0, t_{1244}\right]$} & $\mathcal{C}_{1}(t)$ \\
$C_{42}$ & $2 \eta$ & $B+A^{\prime}$ & {$\left[\pi-\alpha-\frac{\vartheta}{2}, 2(\pi-\alpha)-\vartheta\right]$} & $\mathcal{K}_{2}(t)$ \\
\hline
\end{tabular}

and the excluded area is

$$
\begin{aligned}
& A(\alpha, \vartheta, \eta)=2\left[\frac{1}{2} \operatorname{Sett}(2(1+\eta), 2 \alpha-\vartheta)+\operatorname{Sett}(1+2 \eta, \vartheta)+\operatorname{Sett}\left(2 \eta, \pi-\vartheta-t_{4412}\right)+\operatorname{Sett}\left(1+2 \eta, t_{1244}\right)\right. \\
& \left.+\frac{1}{2} \operatorname{Sett}(2 \eta, 2(\pi-\alpha)-\vartheta)+\mathcal{T}\left(h_{1}, b_{1}, b_{2}\right)+\mathcal{T}\left(h_{2}^{\prime}, b_{2}, b_{3}^{\prime}\right)+\mathcal{T}\left(h_{3}^{\prime \prime}, b_{3}^{\prime}, b_{5}\right)\right] . \\
& \text { 2. } \vartheta \in\left[\vartheta_{4412}, 2(\pi-\alpha)\right]
\end{aligned}
$$

The essential boundary is formed by the arcs

\begin{tabular}{|l|l|l|l|l|}
\hline arc & radius & center & $t$ & base curve \\
\hline$C_{11}$ & $2(1+\eta)$ & $O$ & {$\left[0, \alpha-\frac{\vartheta}{2}\right]$} & $\mathcal{K}_{1}(t)$ \\
$C_{14}$ & $1+2 \eta$ & $B^{\prime}$ & {$\left[t_{1412}, \vartheta\right]$} & $\mathcal{C}_{1}(t)$ \\
$C_{12}$ & $1+2 \eta$ & $A^{\prime}$ & {$\left[0, t_{1214}\right]$} & $\mathcal{C}_{1}(t)$ \\
$C_{42}$ & $2 \eta$ & $B+A^{\prime}$ & {$\left[\pi-\alpha-\frac{\vartheta}{2}, 2(\pi-\alpha)-\vartheta\right]$} & $\mathcal{K}_{2}(t)$ \\
\hline
\end{tabular}

and the excluded area is

$$
\begin{aligned}
& A(\alpha, \vartheta, \eta)=2\left[\frac{1}{2} \operatorname{Sett}(2(1+\eta), 2 \alpha-\vartheta)+\operatorname{Sett}\left(1+2 \eta, \vartheta-t_{1412}\right)+\operatorname{Sett}\left(1+2 \eta, t_{1214}\right)\right. \\
& \left.+\frac{1}{2} \operatorname{Sett}(2 \eta, 2(\pi-\alpha)-\vartheta)+\mathcal{T}\left(h_{1}^{\prime \prime \prime}, b_{1}, b_{2}^{\prime}\right)+\mathcal{T}\left(h_{2}^{\prime \prime}, b_{2}^{\prime}, b_{5}\right)\right] \text {. } \\
& \text { 3. } \vartheta \in[2(\pi-\alpha), \pi]
\end{aligned}
$$

The essential boundary is formed by the arcs

\begin{tabular}{|l|l|l|l|l|}
\hline arc & radius & center & $t$ & base curve \\
\hline$C_{11}$ & $2(1+\eta)$ & $O$ & {$\left[0, \alpha-\frac{\vartheta}{2}\right]$} & $\mathcal{K}_{1}(t)$ \\
$C_{14}$ & $1+2 \eta$ & $B^{\prime}$ & {$\left[t_{1412}, \vartheta\right]$} & $\mathcal{C}_{1}(t)$ \\
$C_{12}^{\text {bis }}$ & $1+2 \eta$ & $A^{\prime}$ & {$\left[0, t_{1214}^{\text {bis }}\right]$} & $\mathcal{K}_{2}(t)$ \\
$C_{11}^{\text {bis }}$ & $2(1+\eta)$ & $O$ & {$\left[\frac{\vartheta}{2}-(\pi-\alpha), \vartheta-2(\pi-\alpha)\right]$} & $\mathcal{C}_{1}(t)$ \\
\hline
\end{tabular}

and the excluded area is

$$
\begin{aligned}
A(\alpha, \vartheta, \eta)= & 2\left[\frac{1}{2} \operatorname{Sett}(2(1+\eta), 2 \alpha-\vartheta)+\operatorname{Sett}\left(1+2 \eta, \vartheta-t_{1412}\right)+\operatorname{Sett}\left(1+2 \eta, t_{1214}^{\mathrm{bis}}\right)\right. \\
& \left.+\frac{1}{2} \operatorname{Sett}(2(1+\eta), \vartheta-2(\pi-\alpha))+\mathcal{T}\left(h_{1}^{\prime \prime \prime}, b_{1}, b_{2}^{\prime}\right)+\mathcal{T}\left(h_{2}^{\prime \prime \prime}, b_{2}^{\prime}, b_{3}^{\prime \prime}\right)\right] .
\end{aligned}
$$

\section{RESULTS}

By joining the expressions given in equations (III.16), (III.19)—or (III.30), if $\alpha \in\left[\frac{\pi}{4}, \frac{\pi}{2}\right]$-(III.20), (III.23), and (III.26) we obtain a piecewise expression for the area $A(\alpha, \vartheta, \eta)$ enclosed by the Minkowski sum $\mathcal{M} \oplus \mathcal{M}^{\prime}$. By Theorem II.1 and since we are in a planar setting, $A(\alpha, \vartheta, \eta)$ also represents the excluded area between the same molecules 
when they are at a relative angle $\varphi:=\pi-\vartheta$. Figure 8 illustrates the behaviour of the excluded area against $\varphi$, when $\eta=10^{-2}$, and $\alpha=\pi / 6$ and $\pi / 3$, respectively. The graphs clearly indicate that the excluded area attains its absolute minimum when the molecules are antiparallel, while the configuration at which the molecules are parallel is only a local minimum. The two minima are separated by a maximum attained at $\varphi \gtrsim \frac{\pi}{2}$. Thus we can say that steric interactions are polar since they distinguish between parallel and antiparallel alignments. Moreover, the fact that the excluded area is minimized when two boomerangs are antiparallel suggests the possibility that an ensemble made of these molecules could exhibit a rich variety of phase transitions: hence, it would be interesting to use the excluded area found here to build a steric interaction potential in the spirit of [16] or [13]. In particular, if a scaled particle approach is followed, the excluded area has to be modified to account the different size of the interacting molecules.

Figure 10 shows the excluded area for a pair of horseshoes against the relative orientation $\varphi=\pi-\vartheta$, for four values of $\alpha$, one for each regime considered here. At variance with boomerangs, the difference between the relative minimum at $\varphi=0$ and the absolute maximum decreases when the opening angle approaches its upper bound $\alpha_{M}$. This is not surprising since, when $\alpha$ is close to $\alpha_{M}$, the molecular morphology hinders the exploration of the concave regions when the molecules are antiparallel. Equivalently, when $\alpha \rightarrow \alpha_{M}$ the molecules tend to acquire an equatorial symmetry that makes parallel and antiparallel configurations undistinguishable.

Following [17], to measure the relative magnitude of the minima of the excluded area, we introduce the polarity index $\varrho$ defined according to

$$
\varrho(\eta, \alpha):=3 \frac{A(\alpha, 0, \eta)-A(\alpha, \pi, \eta)}{A(\alpha, 0, \eta)+A(\alpha, \pi, \eta)}
$$

where the second argument in $A$ is the value of $\varphi$ and the factor 3 has been chosen to normalize the maximum value of $\varrho$ at 1 : in the parlance of $[17], \varrho(\eta, \alpha)=I_{p}(0)$.

Figure 11 shows the behaviour of $\varrho$ as a function of $\alpha \in\left[0, \alpha_{M}\right]$, when $\eta=10^{-2}$. As a result, the polarity index does not have a monotonic profile. For boomerangs $\left(\alpha \leq \frac{\pi}{2}\right)$ the polarity index steadily increases from the value $\varrho(0)=0$ attained for discorectangles that are centro-symmetric molecules. For horseshoes $\varrho$ fails to be a monotone function of $\alpha$ but decreases as $\alpha$ approaches $\alpha_{M}$, attaining the asymptotic value $\varrho\left(\alpha_{M}\right)=0$ in the limit when $\eta \rightarrow 0$, so that a horseshoe reduces to a circle with no shape polarity. Finally, the absolute maximum of $\varrho$ saturates at 1 in the limit when $\eta \ll 1$.

The analytic piecewise expression of the excluded area for a pair of boomerangs or horseshoes could be difficult to handle. However, the function $A_{0}$ defined in equation (III.29) represents a good approximation in the limit when $\eta \ll 1$ and so the molecules are slender bodies. Figure 9 shows the graphs of both $A\left(0.1, \varphi, \frac{\pi}{6}\right)$ and $A_{0}\left(0.1, \varphi, \frac{\pi}{6}\right)$. Although $\eta$ is not exceedingly small, $A_{0}$ is still able to capture the essential behaviour of the excluded area. When $\eta=10^{-2}$, the two graphs are practically identical. We also note that, at this level of approximation, $\eta$ and $\varphi$ are weakly coupled through the angle $\vartheta_{a}(\alpha, \eta)$. In particular, a pair of thin boomerangs and a pair of genuine arcs with $\eta \equiv 0$ have excluded areas with the same anisotropic part.

\section{CONCLUSIONS}

We computed analytically the excluded area for a pair of planar, identical molecules that are a generalization of the discorectangles considered in [16]. The molecules, named boomerangs or horseshoes, depending on the value of an angular parameter, have two important geometric properties. They are not convex and they lack a center of symmetry. The former feature makes the explicit computation of the excluded area a non-trivial task that was overcome by resorting to techniques employed in CAGD that relate the Minkowski sum of two bodies to their convolution curve. Obtaining the convolution curve requires tracking the behaviour of the Gauss map of the two bodies while extracting information on the Minkowski boundary from the profile of the convolution curve requires a trimming procedure for which at the moment there is no analytic general procedure. We managed to succeed by using a molecular profile formed by piecewise circular arcs.

Lack of central symmetry in the molecules makes it possible to explore shape polarity effects on the excluded area. Precisely, it turns out that the absolute minimum of the excluded area as a function of the relative orientation between the two molecules is attained when the molecules are antiparallel, instead of parallel. Following [17], we introduced a polarity index to measure the magnitude of this effect. It turned out that the polarity index attains its absolute maximum when $2 \alpha \gtrsim \pi$ while its absolute minimum 0 is always attained at $\alpha=0$ and also at $\alpha=\alpha_{M}$, when $\eta \rightarrow 0$. This behaviour can be explained by noting that large values of the opening angle mask the concave region bounded by an horseshoe, that cannot be explored by the other molecule. As a result, though the molecules are not centro-symmetric, their asymmetry is hidden.

Clearly this paper opens the ways to several studies. First, it would be interesting to use the analytic expression of the excluded area for a pair of boomerangs or even its approximation (III.29) to explore phase transition by building 
a density-functional theory, in the spirit of [16]. Then, it raises a natural question. Do the techniques employed here work in three space dimensions? By looking again at the CAGD literature ([12], [21]) we gain the impression that, if the molecular shapes are modelled as union of simple patches on which the Gauss map is easily computable, then the task could be successfully pursued, maybe numerically. Another three-dimensional extension that dwells on a different method has been studied in [22] where, by resorting to a mixed approach, both analytical and numerical, we are able to obtain the excluded volume for molecules conceived as aggregates of spheres, generalizing the results of [23] and [24] to different molecular morphologies.

However, also remaining in two space dimensions could be of interest. The unusual behaviour of the excluded area as a function of the relative orientation suggests that a study of an ensemble formed by boomerangs could lead to a rich spectrum of phase transitions, especially when the polarity index is high. A density-functional or a scaled particle theory approach could benefit from the knowledge of the explicit formula for the excluded area as provided in the present paper.

\section{APPENDIX A: ARCS FORMING A BOOMERANG}

We give here the explicit parameterization of the arcs $C_{i j}$ whose properties were listed concisely in the Tables of Sec. III.

$$
C_{11}-O=2(\eta+1)\left[-\sin \left(t-\alpha+\frac{\vartheta}{2}\right) \boldsymbol{e}_{x}+\cos \left(t-\alpha+\frac{\vartheta}{2}\right) \boldsymbol{e}_{y}\right]
$$

in some cases a different base curve was required, leading to the arc

$$
\begin{gathered}
C_{11}^{\mathrm{bis}}-O=2(\eta+1)\left[-\sin \left(t-\alpha-\frac{\vartheta}{2}\right) \boldsymbol{e}_{x}+\cos \left(t-\alpha-\frac{\vartheta}{2}\right) \boldsymbol{e}_{y}\right] . \\
C_{14}-O=B^{\prime}-O+(1+2 \eta)\left[-\sin \left(t-\alpha-\frac{\vartheta}{2}\right) \boldsymbol{e}_{x}+\cos \left(t-\alpha-\frac{\vartheta}{2}\right) \boldsymbol{e}_{y}\right] \\
C_{44}-O=(B-O)+\left(B^{\prime}-O\right)+2 \eta\left[-\sin \left(\pi+t-\alpha+\frac{\vartheta}{2}\right) \boldsymbol{e}_{x}+\cos \left(\pi+t-\alpha+\frac{\vartheta}{2}\right) \boldsymbol{e}_{y}\right] \\
C_{43}-O=(B-O)+(2 \eta-1)\left[-\sin \left(\pi+t-\alpha-\frac{\vartheta}{2}\right) \boldsymbol{e}_{x}+\cos \left(\pi+t-\alpha-\frac{\vartheta}{2}\right) \boldsymbol{e}_{y}\right] \\
C_{42}-O=(B-O)+\left(A^{\prime}-O\right)+2 \eta\left[-\sin \left(t+\alpha+\frac{\vartheta}{2}\right) \boldsymbol{e}_{x}+\cos \left(t+\alpha+\frac{\vartheta}{2}\right) \boldsymbol{e}_{y}\right],
\end{gathered}
$$

where the vectors $A-O, A^{\prime}-O, B-O$, and $B^{\prime}-O$ are given by equations (III.10).

When $\alpha<\pi / 4$, but $\vartheta \in[2 \alpha, \pi-2 \alpha]$, the arc $C_{11}$ is replaced by the arc $C_{24}$ given by

$$
C_{24}-O=(A-O)+\left(B^{\prime}-O\right)+2 \eta\left[-\sin \left(t+\alpha-\frac{\vartheta}{2}\right) \boldsymbol{e}_{x}+\cos \left(t+\alpha-\frac{\vartheta}{2}\right) \boldsymbol{e}_{y}\right],
$$

with base curve given by $\mathcal{C}_{2}$.

When $\vartheta$ exceeds $\pi-2 \alpha$, the arc $C_{12}$ contributes the set $\partial\left(\mathcal{M} \oplus \mathcal{M}^{\prime}\right)$. We take $\mathcal{C}_{1}$ as its base curve, so that

$$
C_{12}-O=\left(A^{\prime}-O\right)+(1+2 \eta)\left[-\sin \left(t-\alpha-\frac{\vartheta}{2}\right) \boldsymbol{e}_{x}+\cos \left(t-\alpha-\frac{\vartheta}{2}\right) \boldsymbol{e}_{y}\right]
$$

When $\mathcal{K}_{2}$ was needed as a base curve, we had to consider the arc

$$
C_{12}^{\mathrm{bis}}-O=\left(A^{\prime}-O\right)+(1+2 \eta)\left[-\sin \left(t+\alpha+\frac{\vartheta}{2}\right) \boldsymbol{e}_{x}+\cos \left(t+\alpha+\frac{\vartheta}{2}\right) \boldsymbol{e}_{y}\right]
$$

While at $t=0 C_{12}(0)$ coincides with $C_{42}(2 \pi-2 \alpha-\vartheta)$, the largest value of $t$ for which $C_{12}$ belongs to $\partial\left(\mathcal{M}_{1} \oplus \mathcal{M}_{2}\right)$ depends on $\vartheta$. In fact, $C_{12}$ intersects $C_{43}$ at a point where $t=t_{1243}$. Correspondingly, $t \in\left[\vartheta, t_{4312}\right] \subseteq[\vartheta, \pi]$ along the portion of the arc $C_{43}$ that contributes to $\partial\left(\mathcal{M} \oplus \mathcal{M}^{\prime}\right)$. When $\vartheta=\vartheta_{1243}$, the whole arc $C_{43}$ lies inside $\partial\left(\mathcal{M} \oplus \mathcal{M}^{\prime}\right)$ 
and $C_{12}$ now crosses the portion of $C_{44}$ belonging to $\partial\left(\mathcal{M} \oplus \mathcal{M}^{\prime}\right)$ at a point where $t=t_{1244}$. Correspondingly, $C_{44}$ belongs to $\partial\left(\mathcal{M} \oplus \mathcal{M}^{\prime}\right)$ only when $t \in\left[t_{4412}, \pi-\vartheta\right]$, for a suitable value $t_{4412}>0$ of $t$. When $\vartheta=\vartheta_{1244}$, the arc $C_{44}$ lies completely in the interior of $\mathcal{M} \oplus \mathcal{M}^{\prime}$. Then, $C_{12}$ crosses $C_{14}$ when $t=t_{1214}$, and the portion of $C_{14}$ in $\partial\left(\mathcal{M} \oplus \mathcal{M}^{\prime}\right)$ satisfies $t \in\left[t_{1412}, 2 \alpha\right]$ We now show how to compute the critical values of $\vartheta$ separating different regimes, together with the values of $t$ at which the different arcs intersect. Let us determine $C_{12} \cap C_{43}$. The values of $t$ at which a point on $C_{12}$ also belongs to $C_{43}$ satisfy the equation

$$
\left|C_{12}(t)-(B-O)\right|^{2}=(2 \eta-1)^{2}
$$

and a straightforward computation based on equations (A.4) and (III.10) shows that the acceptable value of $t$ is

$$
t_{1243}(\eta, \alpha, \theta)=\frac{\vartheta}{2}+\alpha-\arcsin \left[\frac{2 \eta+\sin ^{2}\left(\alpha+\frac{\vartheta}{2}\right)}{(1+2 \eta) \sin \left(\alpha+\frac{\vartheta}{2}\right)}\right]
$$

Similarly, by imposing $\left|C_{43}(t)-\left(A^{\prime}-O\right)\right|^{2}=(2 \eta+1)^{2}$ and using equations (A.3) and (III.10) we arrive at

$$
t_{4312}(\eta, \alpha, \theta)=\frac{\vartheta}{2}+\alpha+\arcsin \left[\frac{2 \eta-\sin ^{2}\left(\alpha+\frac{\vartheta}{2}\right)}{(2 \eta-1) \sin \left(\alpha+\frac{\vartheta}{2}\right)}\right] .
$$

Since $t=\vartheta$ is the smallest admissible value of $t$ on $C_{43}$, to determine the value of $\vartheta_{4312}$ we simply solve the equation $\left|C_{43}(\vartheta)-\left(A^{\prime}-O\right)\right|^{2}=(2 \eta+1)^{2}$ in terms of $\vartheta$. After use of elementary trigonometric identities this requirement is recast as

$$
\frac{2 \eta-1}{2}(\cos 2 \alpha-\cos \vartheta)=2 \eta-\frac{1}{2}[1-\cos (2 \alpha+\vartheta)]
$$

which, after little labour, can be reduced to the form

$$
c_{43} \sin \vartheta+b_{43} \cos \vartheta-a_{43}=0
$$

where

$$
a_{43}(\eta, \alpha):=\frac{1}{2}(2 \eta-1) \cos 2 \alpha+\frac{1}{2}-2 \eta \quad b_{43}(\eta, \alpha):=\frac{1}{2} \cos 2 \alpha+\eta-\frac{1}{2} \quad c_{43}(\alpha)=-\frac{1}{2} \sin 2 \alpha
$$

and that can be solved by setting $x:=\tan \frac{\vartheta}{2}$ and using the identities

$$
\sin \vartheta=\frac{2 x}{1+x^{2}} \quad \cos \vartheta=\frac{1-x^{2}}{1+x^{2}} .
$$

As a result, the acceptable root is

$$
\vartheta_{4312}(\eta, \alpha)=2 \arctan \left(\frac{c_{43}-\sqrt{c_{43}^{2}+b_{43}^{2}-a_{43}^{2}}}{\eta(\cos 2 \alpha-1)}\right)
$$

When $\vartheta>\vartheta_{4312}$, the arc $C_{43}$ no longer contributes to $\partial\left(\mathcal{M} \oplus \mathcal{M}^{\prime}\right)$, and we have to look for $C_{12} \cap C_{44}$. Thus, we first solve

$$
\left|C_{12}(t)-(B-O)-\left(B^{\prime}-O\right)\right|^{2}=4 \eta^{2}
$$

by finding ( see equations (A.4) and (III.10)) the values of $u:=t-\alpha-\frac{\vartheta}{2}$ that obey

$$
a_{44} \sin u-b_{44} \cos u+k_{44}=0
$$

where

$$
a_{44}(\alpha, \vartheta):=2 \sin \left(\alpha+\frac{\vartheta}{2}\right)+\sin \left(\alpha-\frac{\vartheta}{2}\right), \quad b_{44}(\alpha, \vartheta):=\cos \left(\alpha-\frac{\vartheta}{2}\right), \quad k_{44}(\eta, \alpha, \vartheta):=1+\frac{2 c_{44}(\alpha, \vartheta)}{1+2 \eta}
$$

with

$$
c_{44}(\alpha, \vartheta):=2 \sin \left(\alpha+\frac{\vartheta}{2}\right) \sin \alpha \cos \frac{\vartheta}{2}
$$


It is not difficult to verify that the admissible solution of equation (A.10) in terms of $t$ is

$$
t_{1244}(\eta, \alpha, \vartheta)=\alpha+\frac{\vartheta}{2}+2 \arctan \left(\frac{-a_{44}-\sqrt{a_{44}^{2}+b_{44}^{2}-k_{44}^{2}}}{k_{44}+b_{44}}\right)
$$

Correspondingly, by solving $\left|C_{44}(t)-\left(A^{\prime}-O\right)\right|^{2}=(1+2 \eta)^{2}$ and using equations (A.2) and (III.10) we find

$$
t_{4412}(\eta, \alpha, \vartheta)=\alpha-\frac{\vartheta}{2}+2 \arctan \left(\frac{-a_{44}+\sqrt{a_{44}^{2}+b_{44}^{2}-\left(\frac{c_{44}}{\eta}-1\right)^{2}}}{b_{44}+\frac{c_{44}}{\eta}-1}\right) .
$$

Since the largest value of $t$ for which $C_{44}$ is an admissible arc is $t=\pi-\vartheta$, by solving the equation $\mid C_{44}(\pi-\vartheta)-$ $\left.\left(A^{\prime}-O\right)\right|^{2}=(1+2 \eta)^{2}$ in terms of $\vartheta$ we find the upper bound on the values of $\vartheta$ for which the arc $C_{44}$ contributes to $\partial\left(\mathcal{M} \oplus \mathcal{M}^{\prime}\right)$. It turns out that $\vartheta$ has to satisfy the equation

$$
d_{44} \cos \vartheta-e_{44} \sin \vartheta+f_{44}=0
$$

where

$$
d_{44}(\eta, \alpha):=\left(1+\frac{1}{2 \eta}\right)(\cos 2 \alpha-1) \quad e_{44}(\eta, \alpha):=\sin 2 \alpha\left(1+\frac{1}{2 \eta}\right) \quad f_{44}(\eta, \alpha):=\frac{1}{2 \eta}(\cos 2 \alpha-1) .
$$

By resorting to the identities (A.8), it is possible to prove that

$$
\vartheta_{4412}(\eta, \alpha)=2 \arctan \left(\frac{e_{44}+\sqrt{e_{44}^{2}+d_{44}^{2}-f_{44}^{2}}}{f_{44}-d_{44}}\right) .
$$

In the limit when $\eta \rightarrow 0$, both $\vartheta_{4312}$ and $\vartheta_{4412}$ tend to the continuous function

$$
\vartheta_{l}(\alpha):= \begin{cases}\pi & \text { if } \alpha \in\left[0, \frac{\pi}{2}\right] \\ 2(\pi-\alpha) & \text { if } \alpha \in\left[\frac{\pi}{2}, \pi\right]\end{cases}
$$

To study the behaviour of $\vartheta_{4312}$ and $\vartheta_{4412}$ when $\alpha<\frac{\pi}{2}$ we set

$$
\vartheta_{4312}=\pi-\left(\gamma_{1} \eta+\gamma_{2} \eta^{2}\right)
$$

in equation (A.7) and

$$
\vartheta_{4412}=\pi-\left(\lambda_{1} \eta+\lambda_{2} \eta^{2}\right)
$$

in equation (A.10) and expand the resulting equations in powers of $\eta$. By setting the coefficients of different powers of $\eta$ equal to zero we obtain

$$
\gamma_{1}=\lambda_{1}=2 \tan \alpha, \gamma_{2}=-2 \tan ^{3} \alpha, \lambda_{2}=2 \tan \alpha\left(2-\tan ^{2} \alpha\right) .
$$

This approach fails at $\alpha=\frac{\pi}{2}$ where both $\pi-\vartheta_{4312}$ and $\pi-\vartheta_{4412}$ behave like $\sqrt{\eta}$, instead of $\eta$ like in this case. In fact, this is a consequence of the knee that the limit function $\vartheta_{l}(\alpha)$ has at $\alpha=\frac{\pi}{2}$. We limit ourselves to point out this feature, but we do not proceed in the computations.

When $\vartheta>\vartheta_{4412}$ also $C_{44}$ lies in the interior of $\mathcal{M} \oplus \mathcal{M}^{\prime}$ and we have to turn attention to $C_{12} \cap C_{14}$. We first solve

$$
\left|C_{12}(t)-\left(B^{\prime}-O\right)\right|^{2}=(1+2 \eta)^{2}
$$

to determine the value $t_{1214}$ of $t$ at which $C_{12}$ intersects $C_{14}$. It is not difficult to check that

$$
t_{1214}(\eta, \alpha, \vartheta)=\alpha+\vartheta-\pi+\arcsin \left(\frac{1}{1+2 \eta} \sin \alpha\right) \text {. }
$$

Similarly, by use of equations (A.1) and (III.10) we determine the value $t_{1412}$ of $t$ at which $C_{14}$ crosses the arc $C_{12}$ by imposing that

$$
\left|C_{14}(t)-\left(A^{\prime}-O\right)\right|^{2}=(1+2 \eta)^{2}:
$$


it is possible to prove that the admissible root is given by

$$
t_{1412}(\eta, \alpha, \vartheta):=\alpha+\vartheta-\pi-\arcsin \left(\frac{1}{1+2 \eta} \sin \alpha\right)
$$

[1] L. Onsager: The effects of shape on the interaction of colloidal particles, Ann. N. Y. Acad. Sci. 51, 627-659 (1949).

[2] A. Isihara: Determination of molecular shape by osmotic measurement, J. Chem. Phys. 18, 1446-1449 (1950).

[3] T. Kihara: Virial coefficients and models of molecules in gases, Rev. Mod. Phys. 25, 831-843 (1953).

[4] B. Tjipto-Margo, and G.T. Evans: The Onsager theory of the isotropic-nematic liquid-crystal transition: biaxial particles in uniaxial phases, J. Chem. Phys. 94, 4546-4556 (1991).

[5] G.S. Singh, and B. Kumar: Molecular fluids and liquid crystals in convex-body coordinates systems, Ann. Phys. 294, 24-47 (2001).

[6] B. Mulder: Solution of the excluded volume problem for biaxial particles, Liq. Cryst. 1, 539-551 (1986).

[7] B.M. Mulder: The excluded volume of hard sphero-zonotopes, Mol. Phys. 103, 1411-1424 (2005).

[8] P. I. C. Teixeira, A. J. Masters, B. M. Mulder: Biaxial nematic order in the hard-boomerang fluid, Mol. Crys. Liq. Cryst. 323, (1998) 167-189.

[9] I.-K. Lee, M.-S. Kim, G. Elber: Polynomial/rational approximation of Minkowski sum boundary curves, Graph. Mod. Im. Proc. 60 (1998), 136-165.

[10] T. Lozano-Pérez: Spatial planning: a configuration space approach, IEEE Trans. Comp. C-32 (1983), $108-120$.

[11] D. Blackmore, R. Samulyak, M.C. Leu: Trimming swept volumes, Computer-Aided Design 31 (1999), $215-223$.

[12] J. Hass, R.T. Farouki, C.Y. Han, X. Song, T.W. Sederberg: Guaranteed consistency of surface intersections and trimmed surfaces using a coupled topology resolution and domain decomposition scheme, Adv. Comput. Math. 27 (2007), 1-26.

[13] H. Schlacken, H.-J. Mögel, P. Schiller: Orientational transitions of two-dimensional hard rod fluids, Molec. Phys. 93 (1998), $777-787$.

[14] K. Zhao, C. Harrison, D. Huse, W. B. Russel, P. M. Chaikin: Nematic and almost-tetratic phases of colloidal rectangles, Phys. Rev. E 76 (2007), 040401(R).

[15] D.A. Triplett, K.A. Fichthorn: Monte Carlo simulation of two-dimensional hard rectangles: Confinement effects, Phys. Rev. E 77 (2008), 011707.

[16] Y. Martinez-Ratón, E. Velasco, L. Mederos: Effect of particle geometry in two-dimensional liquid crystals, J. Chem. Phys. 122 (2005), 064903.

[17] F. Bisi, G.E. Durand, R. Rosso, E.G. Virga: Polar steric interactions for V-shaped molecules, Phys. Rev. E 011705 (2008).

[18] Y. Lansac, P.K. Maiti, N.A. Clark, M.A. Glaser: Phase behaviour of bent-core molecules, Phys. Rev. E 67 (2003), 011703.

[19] C.L. Bajaj, M.-S. Kim: Generation of configuration space obstacles: the case of moving algebraic curves, Algorithmica 4 (1989), 157-172.

[20] M. Peternell, T. Steiner: Minkowski sum boundary surfaces of 3D-objects, Graph. Mod. 69 (2007), 180-190.

[21] J. Gravesen: Surfaces parametrized by the normals, Computing 79, 175-183 (2007).

[22] F. Bisi, R. Rosso: Excluded volume for molecules formed by aggregates of spheres, in preparation (2008).

[23] D.C. Williamson, G. Jackson: Excluded volume for a pair of linear chains of tangent hard spheres with an arbitrary relative orientation, Molec. Phys. 86 (1995), 819-836.

[24] K.M. Jaffer, S.B. Opps, D.E. Sullivan: The nematic-isotropic phase transition in linear fused hard sphere chain fluids, J. Chem. Phys. 110 (1999), 11630-11642. 

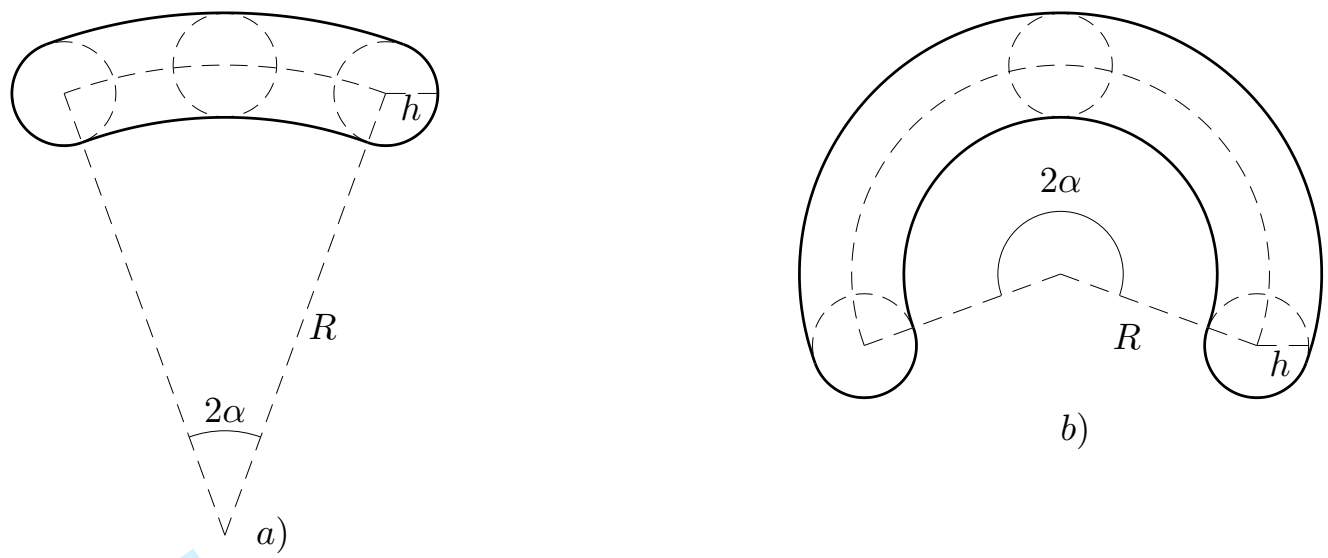

b)

FIG. 1: Sketch of: $a$ ) a boomerang and $b$ ) a horseshoe. The molecules differ because the angle $\alpha$ is less than $\frac{\pi}{2}$ in the former case and larger than $\pi / 2$ in the latter. Apart from this, both molecules can be obtained by moving the center of a disk of radius $h$, dashed in both figures, along the dashed circular arc of radius $R$.

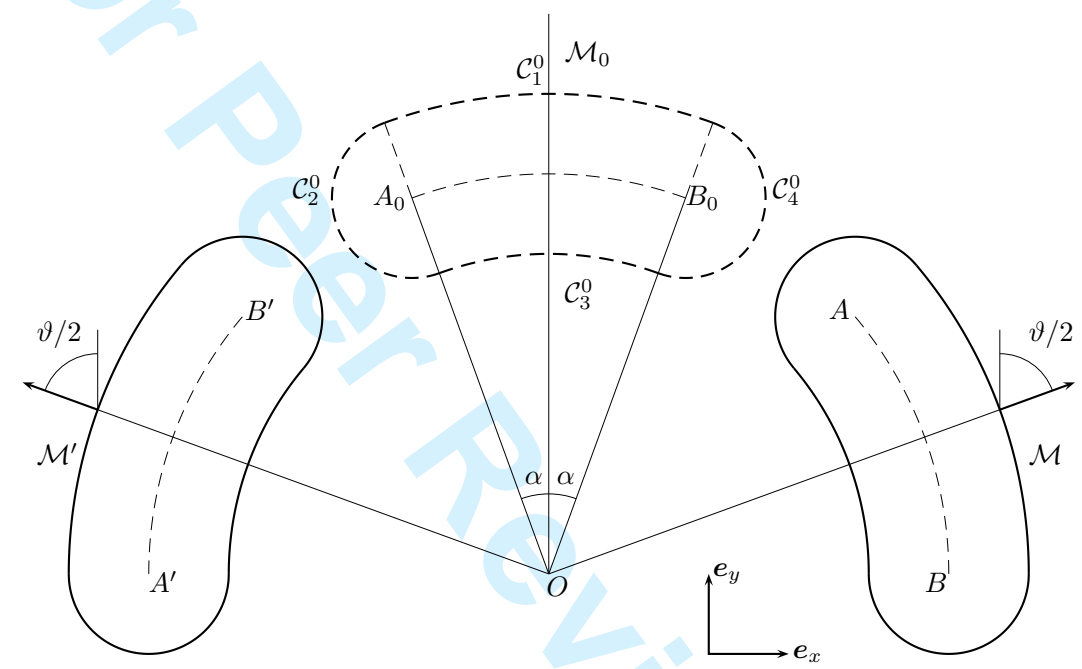

FIG. 2: The molecules $\mathcal{M}$ and $\mathcal{M}^{\prime}$ are obtained by rotating the referential molecule $\mathcal{M}_{0}$ about the axis $\boldsymbol{e}_{z}=\boldsymbol{e}_{x} \times \boldsymbol{e}_{y}$ passing through the point $O$ by an angle $-\vartheta / 2$ and $\vartheta / 2$, respectively. Details of the molecular morphology are explained in the text. 


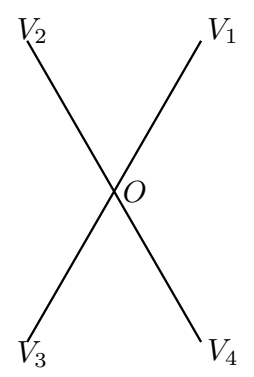

a)

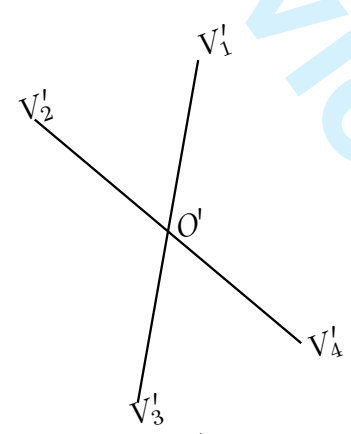

b)

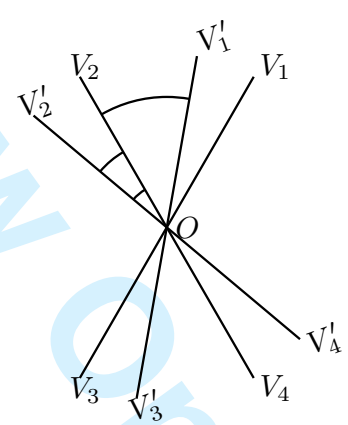

c)

FIG. 4: The cross in $a$ ) schematically represents the images of the Gauss maps along the arcs of $\mathcal{M}$. The Gauss map $\mathcal{N}\left(\mathcal{C}_{1}\right)$ spans the angle $\angle V_{1} O V_{2} ; \mathcal{N}\left(\mathcal{C}_{2}\right)$ spans the $\pi$-angle $\angle V_{2} O V_{4} ; \mathcal{N}\left(\mathcal{C}_{3}\right)$ spans (clockwise) the angle $\angle V_{4} O V_{3}$ and $\mathcal{N}\left(\mathcal{C}_{4}\right)$ spans the $\pi$-angle $\angle V_{1} O V_{3}$. The cross in $b$ ) conveys the same information for $\mathcal{M}^{\prime}$ and so the Gauss maps $\mathcal{N}\left(\mathcal{K}_{1}\right), \ldots, \mathcal{N}\left(\mathcal{K}_{4}\right)$ span, respectively, the angles $\angle V_{1}^{\prime} O^{\prime} V_{2}^{\prime}, \angle V_{2}^{\prime} O^{\prime} V_{4}^{\prime}, \angle V_{4}^{\prime} O V_{3}^{\prime} \angle V_{1}^{\prime} O^{\prime} V_{3}^{\prime}$. Finally, in $c$ ), the two crosses are superimposed to see the arcs along which the Gauss maps coincide: this happens, for instance, along $\angle V_{1}^{\prime} O V_{2}$ for the arcs $\mathcal{C}_{1}$ and $\mathcal{K}_{1}$, and along $\angle V_{2} O V_{2}^{\prime}$ for the $\operatorname{arcs} \mathcal{C}_{2}$ and $\mathcal{K}_{1}$. We note that this scenario holds when $\alpha \in\left[0, \frac{\pi}{4}\right]$ and $\vartheta \in[0,2 \alpha]$. 

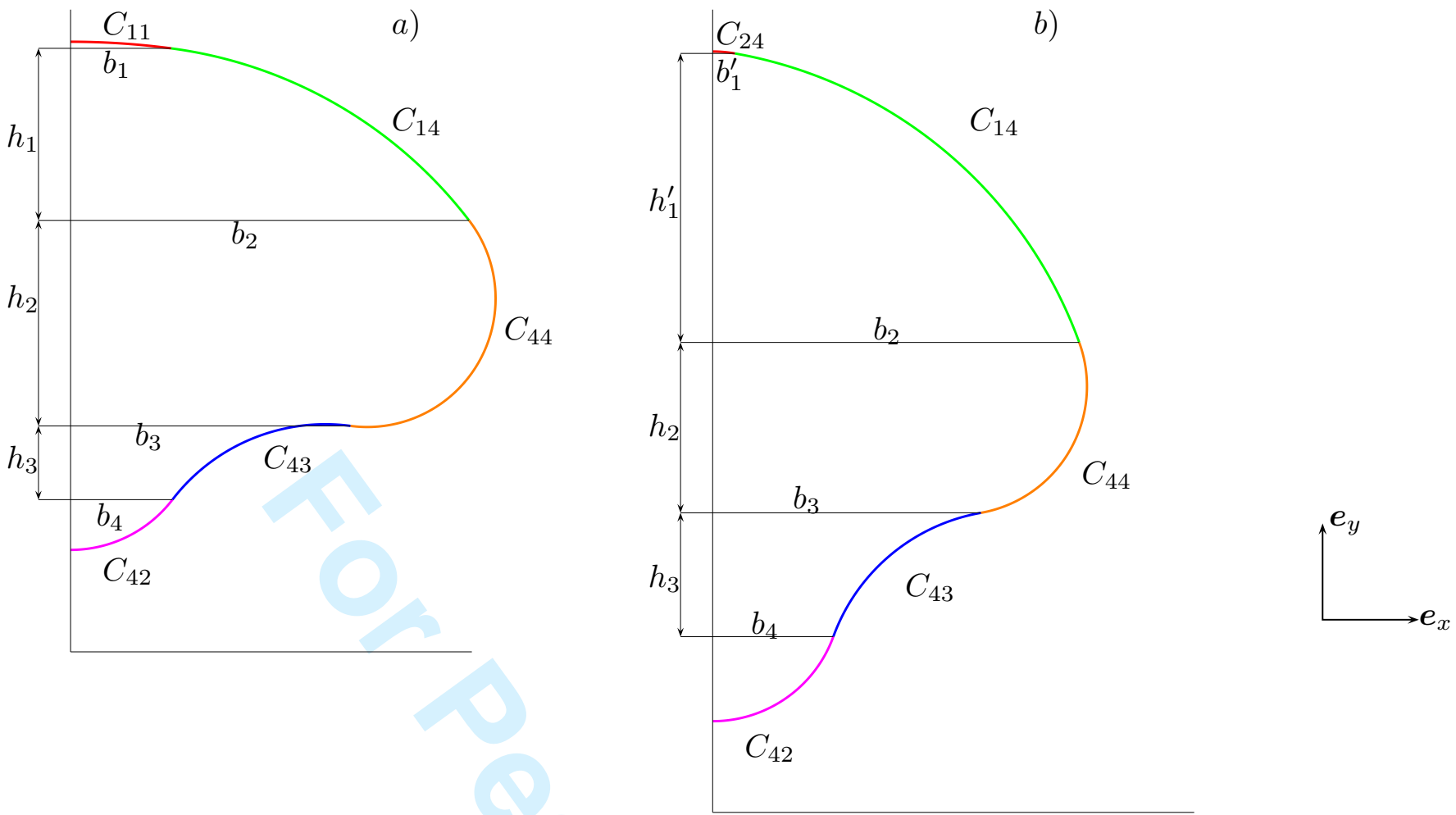

FIG. 5: a): The reduced set $\mathcal{R}_{\oplus}$ corresponding to the values $\alpha=\pi / 6$ and $\vartheta=\pi / 4 \in[0,2 \alpha]$. The excluded region is obtained by pasting to $\mathcal{R}_{\oplus}$ its symmetric set with respect to the $y$-axis. The excluded area is given by equations III.13 and III.16. $b$ ) The reduced set $\mathcal{R}_{\oplus}$ corresponding to the values $\alpha=\pi / 6$ and $\vartheta=8 \pi / 18 \in[2 \alpha, \pi-2 \alpha]$. Compared to $a$ ), the arc $\mathcal{C}_{11}$ is replaced by the $\operatorname{arc} \mathcal{C}_{42}$, while the $\operatorname{arc} \mathcal{C}_{14}$ spans the interval $t \in[0,2 \alpha]$. The excluded area is now given by equations (III.17) and (III.19). 
1

2

3

4

5

6

7

8

9

10

11

12

13

14

15

16

17

18

19

20

21
FIG. 6: The reduced set $\mathcal{R}_{\oplus}$ corresponding to the values $\alpha=\pi / 6$ and $\vartheta=13 \pi / 18 \in\left[\pi-2 \alpha, \vartheta_{4312}\right]$. The admissible arcs $\mathcal{C}_{43}$ an $\mathcal{C}_{12}$ do not join at their end-points and so their intersection is needed to obtain a correct expression for the excluded area. The dashed line is the portion of $\mathcal{C}_{12}$ that lies within the boundary of $\mathcal{M} \oplus \mathcal{M}^{\prime}$. The excluded area is given by equations (III.20) and, when $\eta \ll 1$, (III.22). 

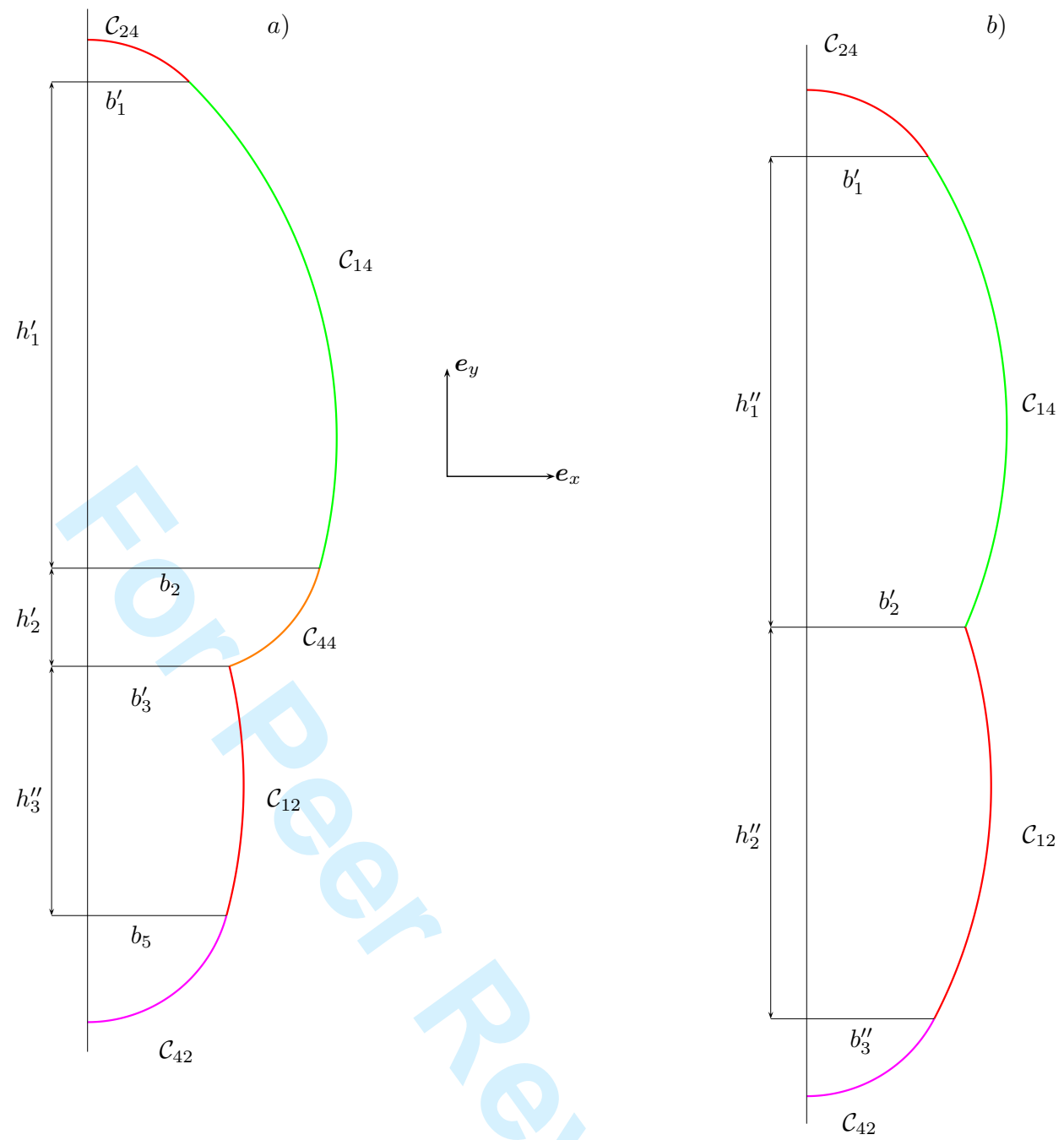

FIG. 7: $a$ ): The reduced set $\mathcal{R}_{\oplus}$ corresponding to the values $\alpha=\pi / 6$ and $\vartheta=15 \pi / 18 \in\left[\vartheta_{4312}, \vartheta_{4412}\right]$. The arc $\mathcal{C}_{43}$ lies in the interior of $\mathcal{R}_{\oplus}$ and $\mathcal{C}_{12}$ intersects $\mathcal{C}_{44}$. The excluded area is now given by equation (III.23). When $\eta \ll 1$ this case disappears. b): The reduced set $\mathcal{R}_{\oplus}$ corresponding to the values $\alpha=\pi / 6$ and $\vartheta=17.5 \pi / 18 \in\left[\vartheta_{4412}, \pi\right]$. Also the arc $\mathcal{C}_{44}$ lies in the interior of $\mathcal{R}_{\oplus}$ and $\mathcal{C}_{12}$ intersects $\mathcal{C}_{14}$. On approaching $\vartheta=\pi$ the set $\mathcal{R}_{\oplus}$ also acquires an equatorial symmetry. The excluded area is given by equation (III.26) and, when $\eta \ll 1$, (III.28). 


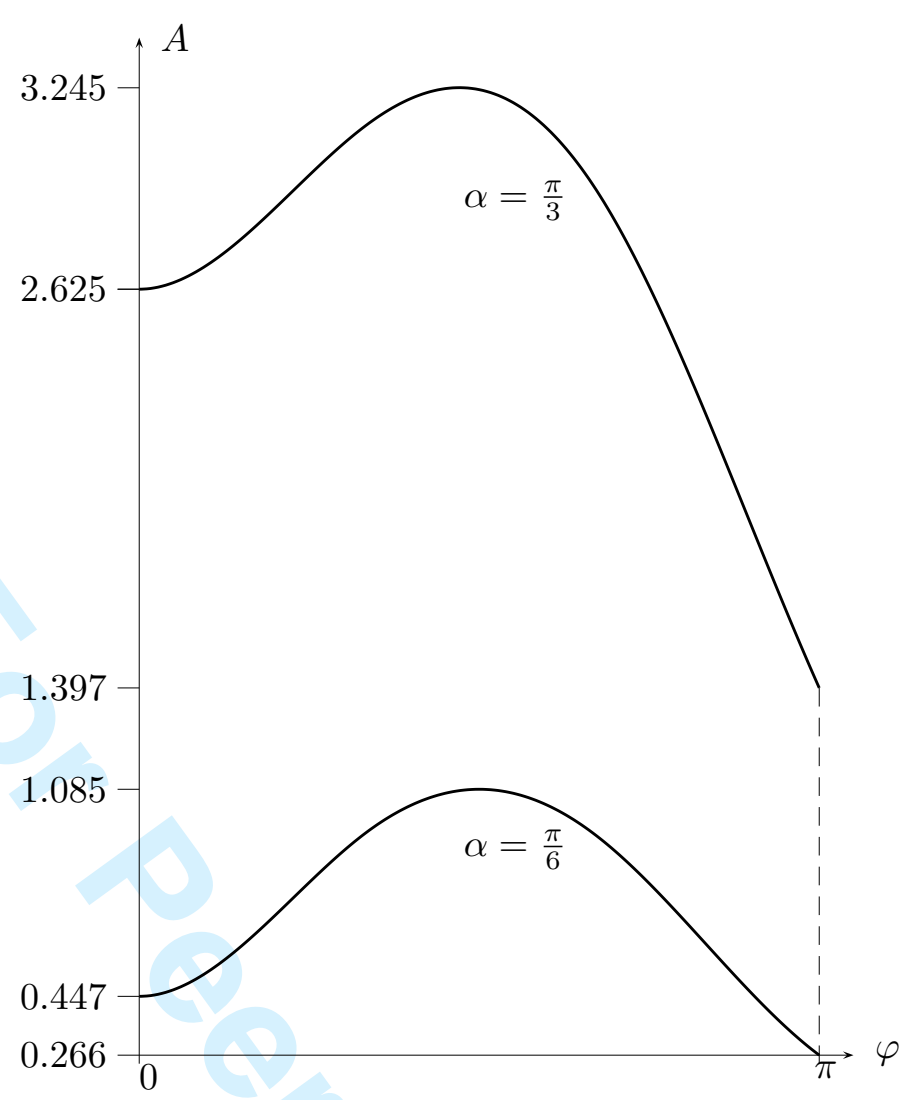

FIG. 8: The excluded area $A(\alpha, \varphi, \eta)$ between two boomerangs is plotted against their relative orientation $\varphi=\pi-\vartheta$, when $\eta=10^{-2}$ and $\alpha=\frac{\pi}{6}$ or $\frac{\pi}{3}$. In both cases $A$ attains its absolute minimum at $\varphi=\pi$, that is, when the molecules are antiparallel. The configuration at which molecules are parallel is still a local minimum of $A$. The two minima are separated by a maximum that is attained when $\varphi$ is close to $\frac{\pi}{2}$. 


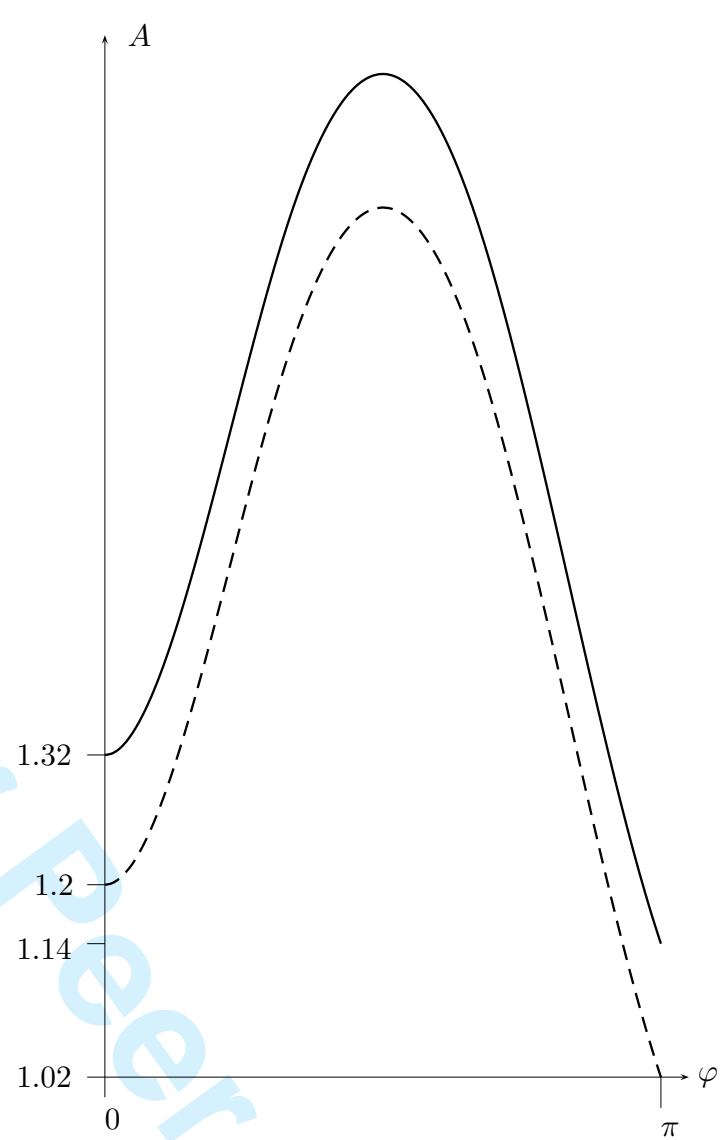

FIG. 9: The graphs of the exact excluded area $A(\alpha, \varphi, \eta)$ (solid line) and of its approximation $A_{0}(\alpha, \varphi, \eta)$ (dashed line) are shown for $\eta=10^{-1}$ and $\alpha=\frac{\pi}{6}$. Although there is a quantitative discrepancy, also for such values of $\eta A_{0}$ follows precisely the qualitative details of $A$. Decreasing $\eta$ makes the quantitative agreement satisfactory and when $\eta \approx 10^{-2}$ the graphs of $A$ and $A_{0}$ practically coalesce. 


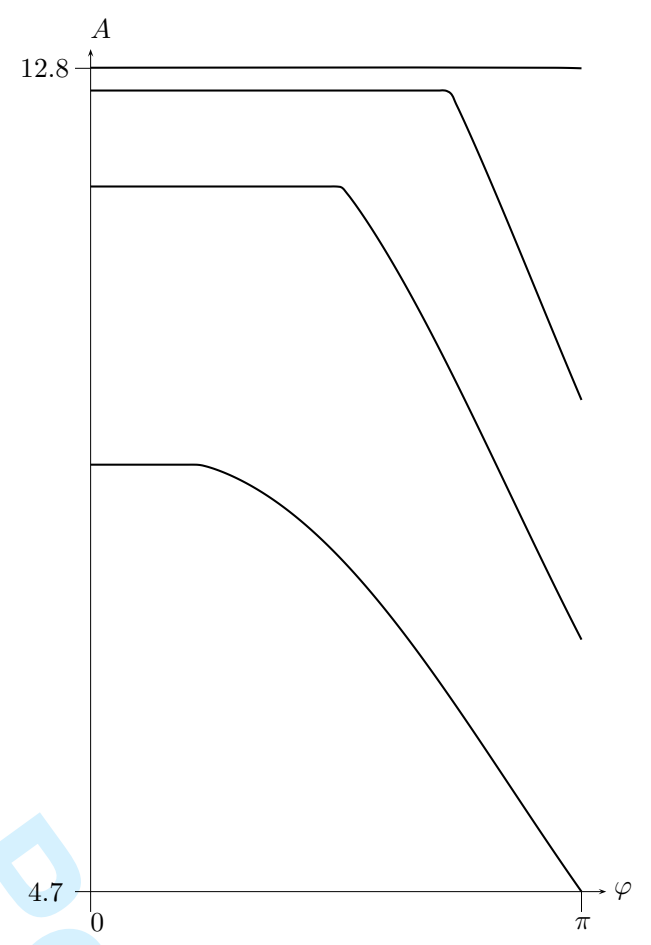

FIG. 10: The dimensionless excluded area $A(\alpha, \varphi, \eta)$ has been plotted against the relative orientation $\varphi=\pi-\vartheta$ when $\eta=0.01$, for different values of $\alpha$ that, from bottom to top, bisect each of the intervals $\left[\frac{\pi}{2}, \alpha_{\mathrm{cr}}\right],\left[\alpha_{\mathrm{cr}}, \alpha_{\mathrm{cr}}^{*}\right],\left[\alpha_{\mathrm{cr}}^{*}, \alpha_{0}\right]$, and $\left[\alpha_{0}, \alpha_{M}\right]$. All profiles have the same qualitative behaviour: the excluded area has a plateau that becomes larger and larger when $\alpha$ increases, and then attains it absolute minimum at $\varphi=\pi$. On increasing $\alpha$, the local minimum at $\varphi=0$ becomes shallower and shallower. We note that the discontinuity at $\varphi=\pi-\vartheta_{1244}^{*}$ cannot be appreciated on this scale. 


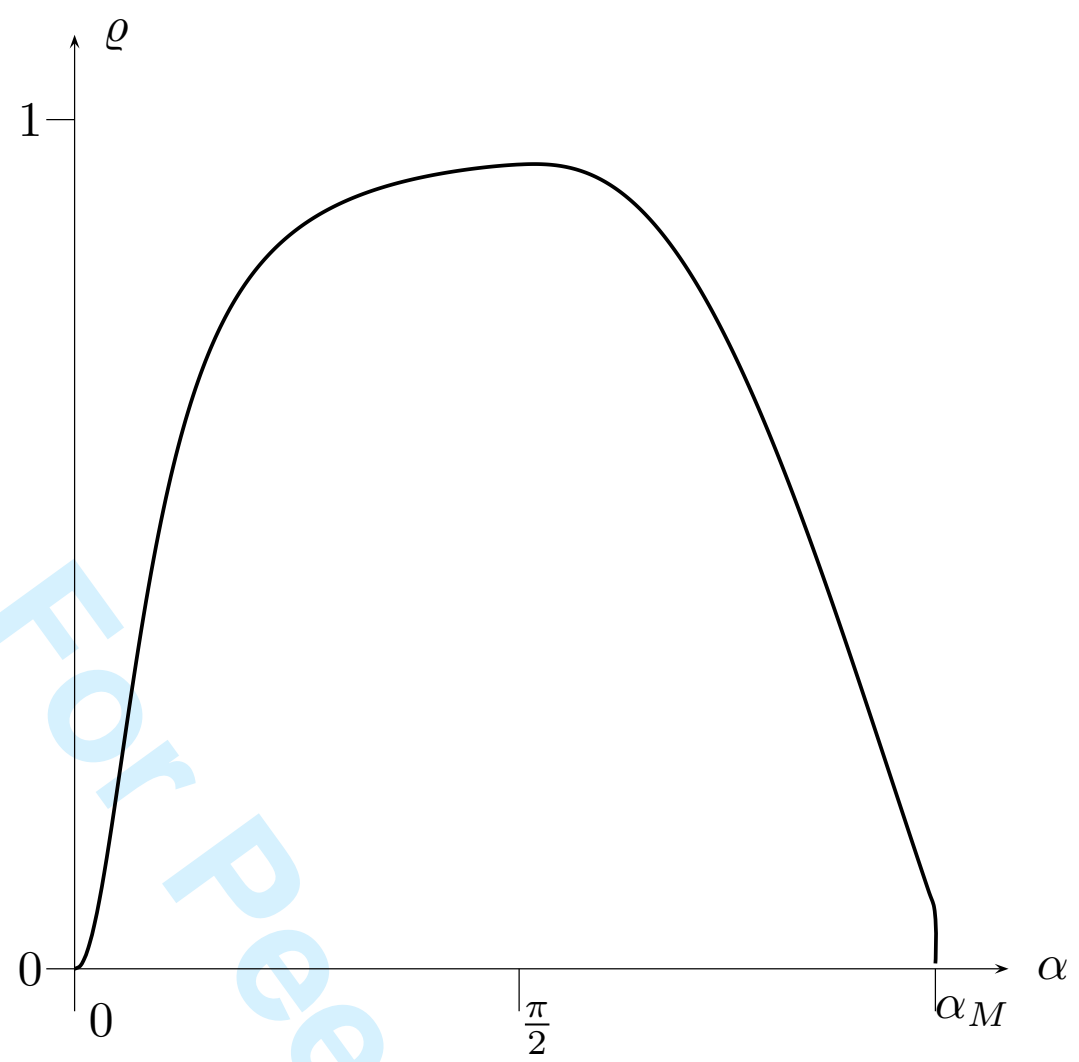

FIG. 11: The polarity index $\varrho$ defined in equation (IV.1) is plotted against $\alpha \in\left[0, \alpha_{M}\right]$, when and $\eta=10^{-2}$. As a general rule, $\varrho$ attains its maximum when $\alpha$ is slightly larger than $\frac{\pi}{2}$. On varying $\eta$, no qualitative changes occur in the graph of $\varrho$ whose maximum saturates at 1 when $\eta \rightarrow 0$. 
FIG. 12: When $\vartheta=\vartheta_{1244}^{*}$, the arcs $C_{12}$ and $C_{44}$ are tangent in $T$. The arc $C_{43}$, bounded by the points $P$ and $Q$ is trapped in the interior of $\mathcal{R}_{\oplus}$ and does not contribute to its boundary. This has also an effect on the excluded area that suffers a tiny jump at $\vartheta=\vartheta_{1244}^{*}$ since the area bounded by the closed contour $T Q P$ is not zero.

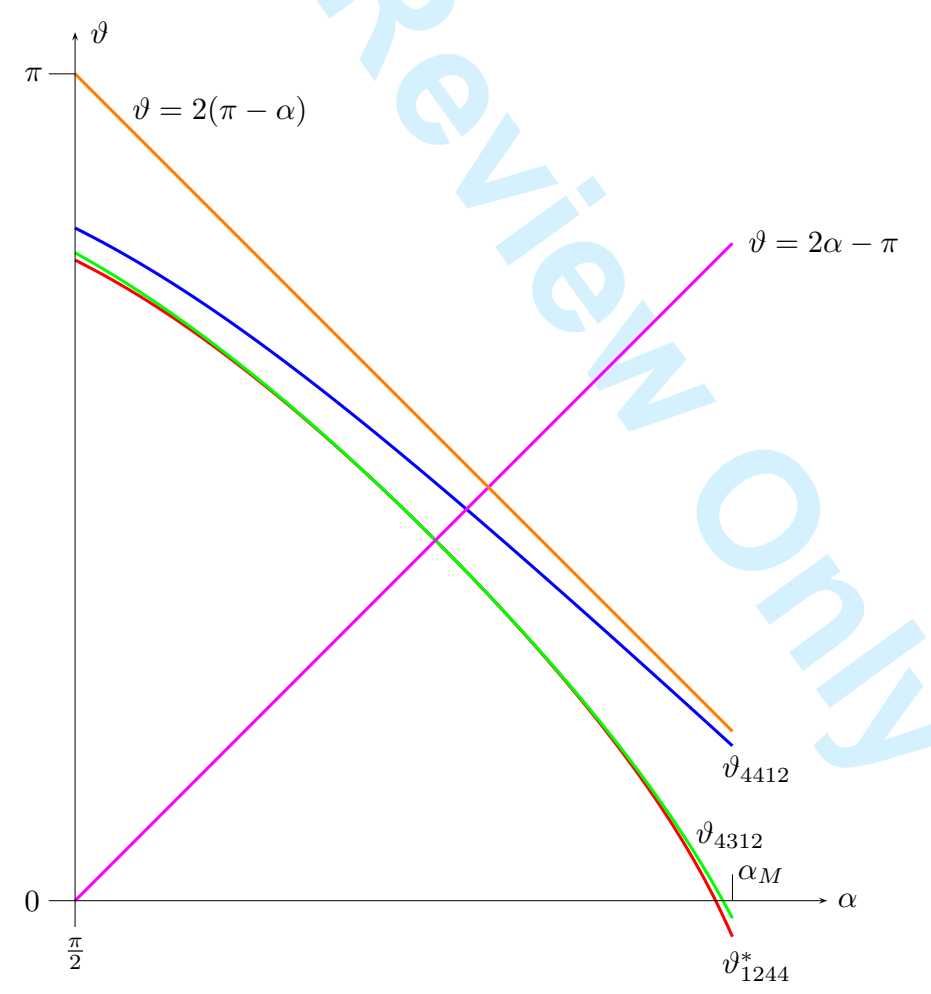

FIG. 13: The functions $\vartheta_{1244}^{*}$ (red curve), $\vartheta_{4312}$ (green), $\vartheta_{4412}$ (blue) are plotted against $\alpha \in\left[\frac{\pi}{2}, \alpha_{M}\right]$ when $\eta=0.1$. The straight lines are $\vartheta=2 \alpha-\pi$ (magenta) and $\vartheta=2(\pi-\alpha)$ (orange). 

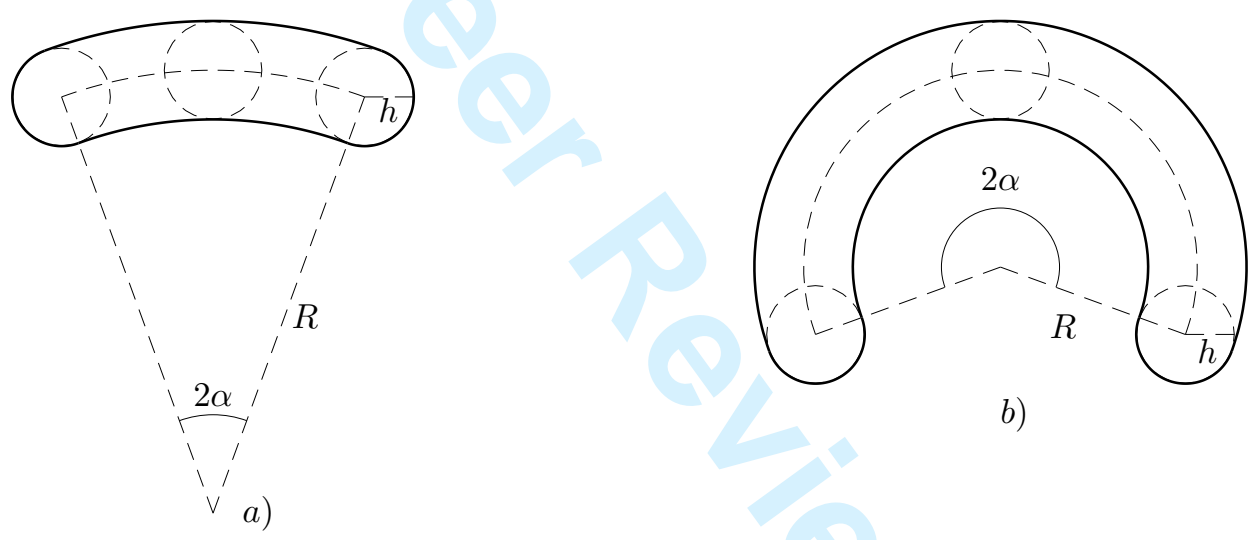

b) 


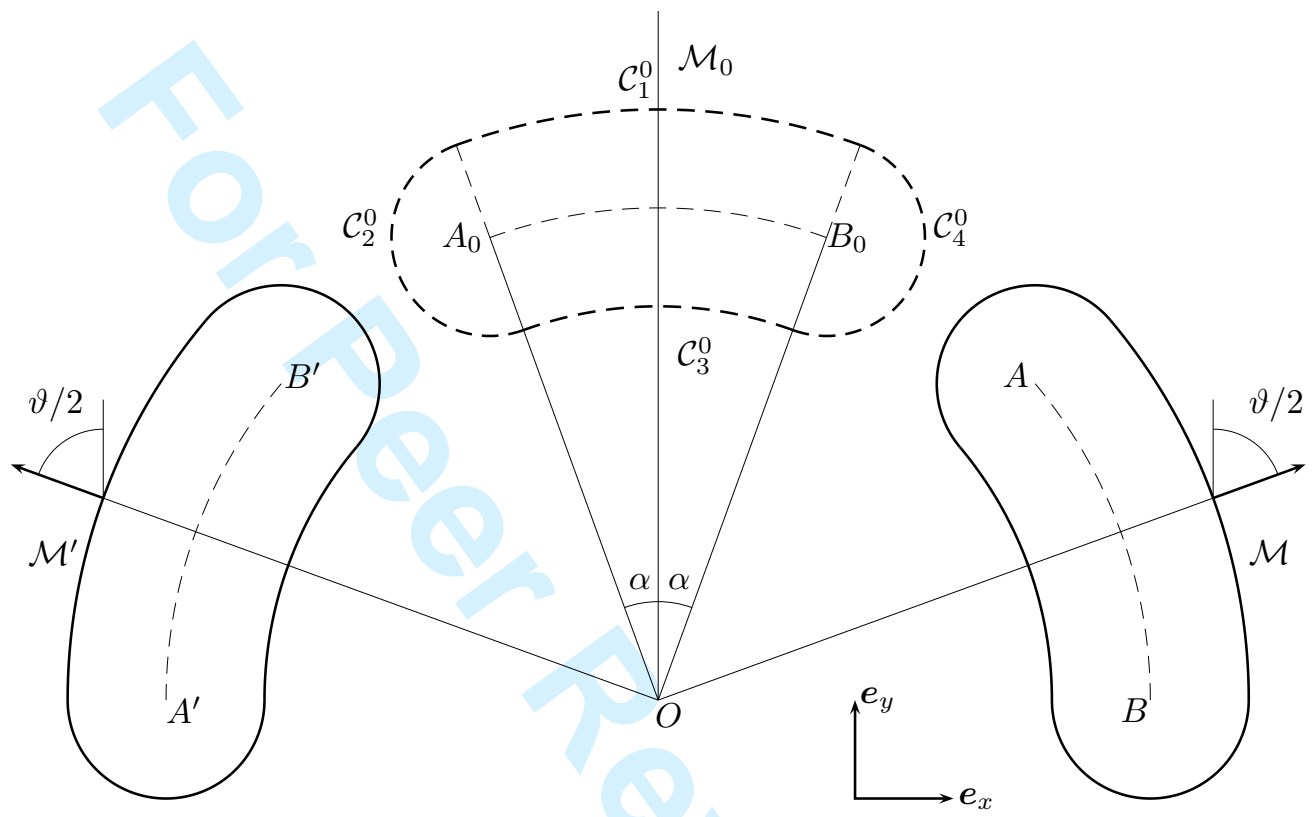




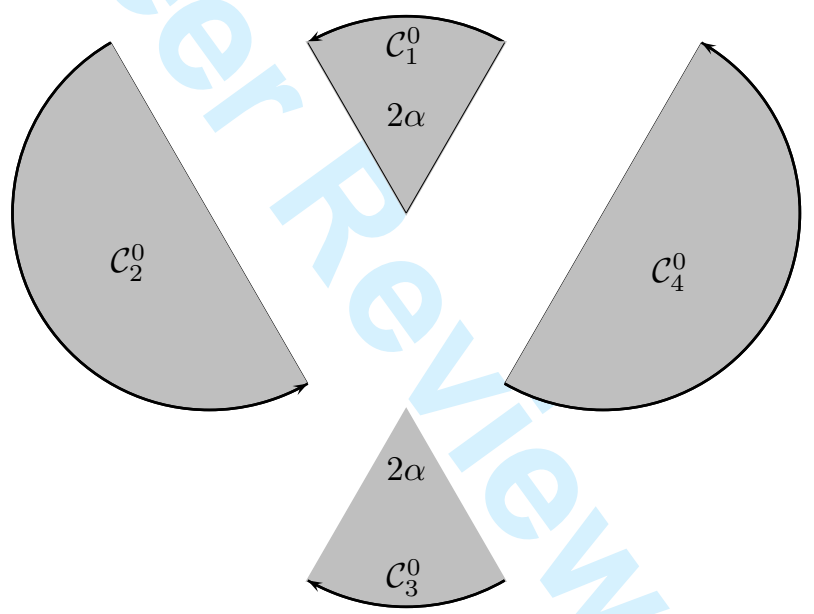




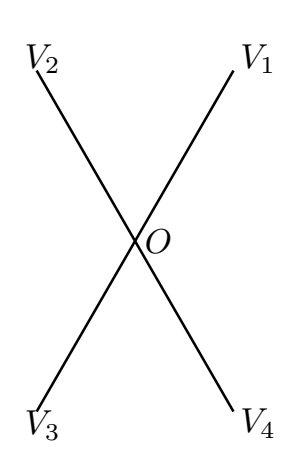

a)

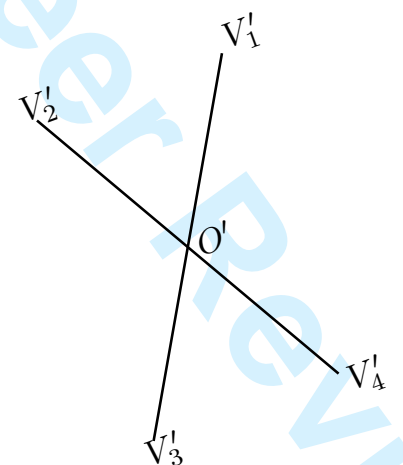

b)

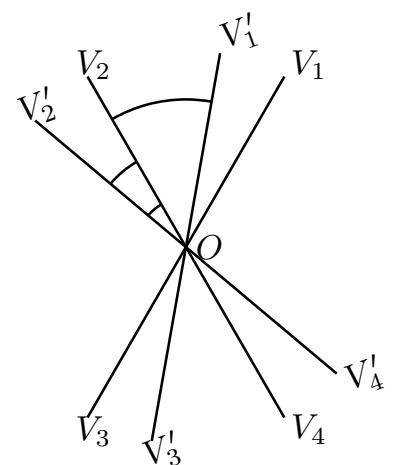

c) 

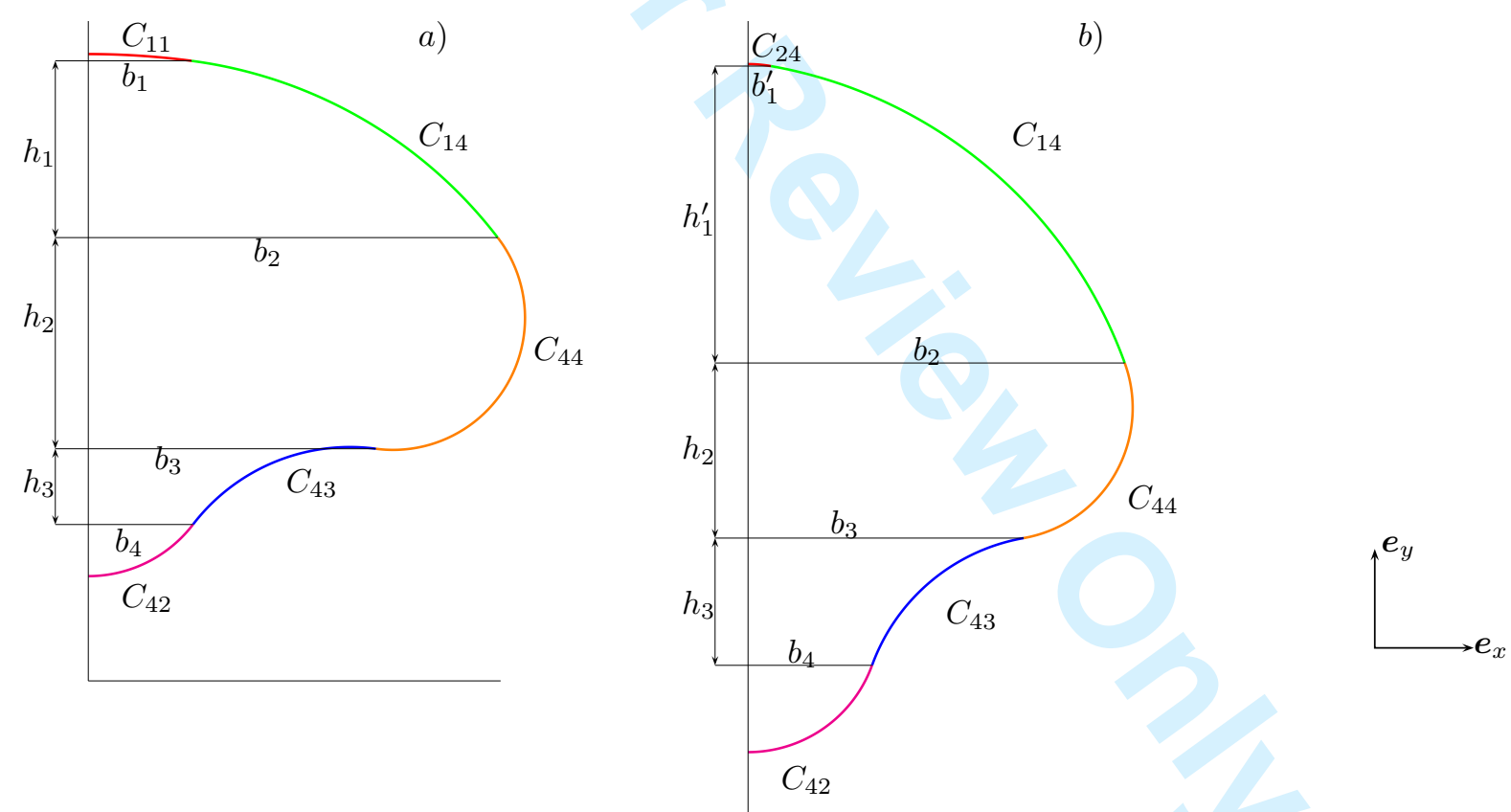

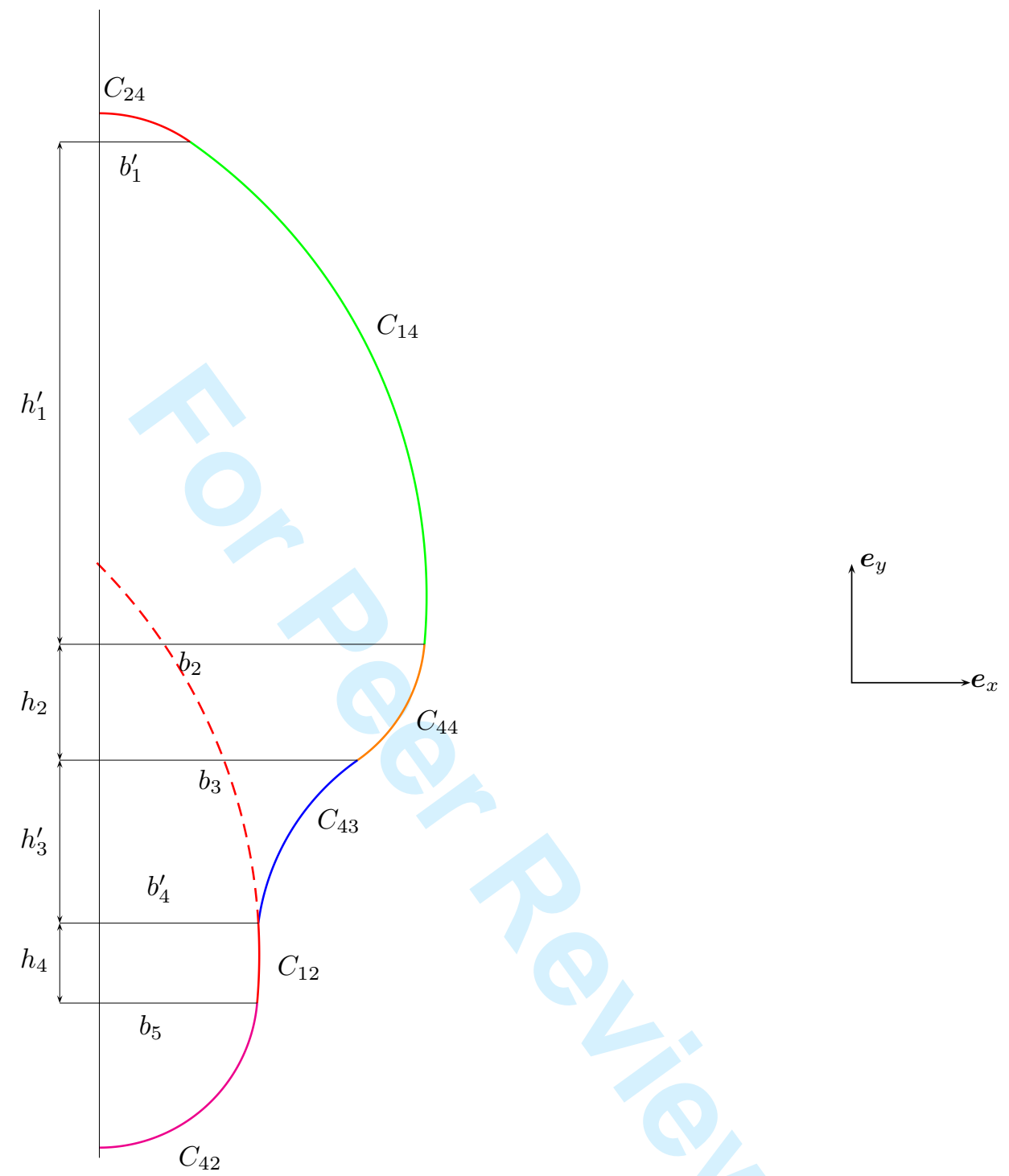

40

41 


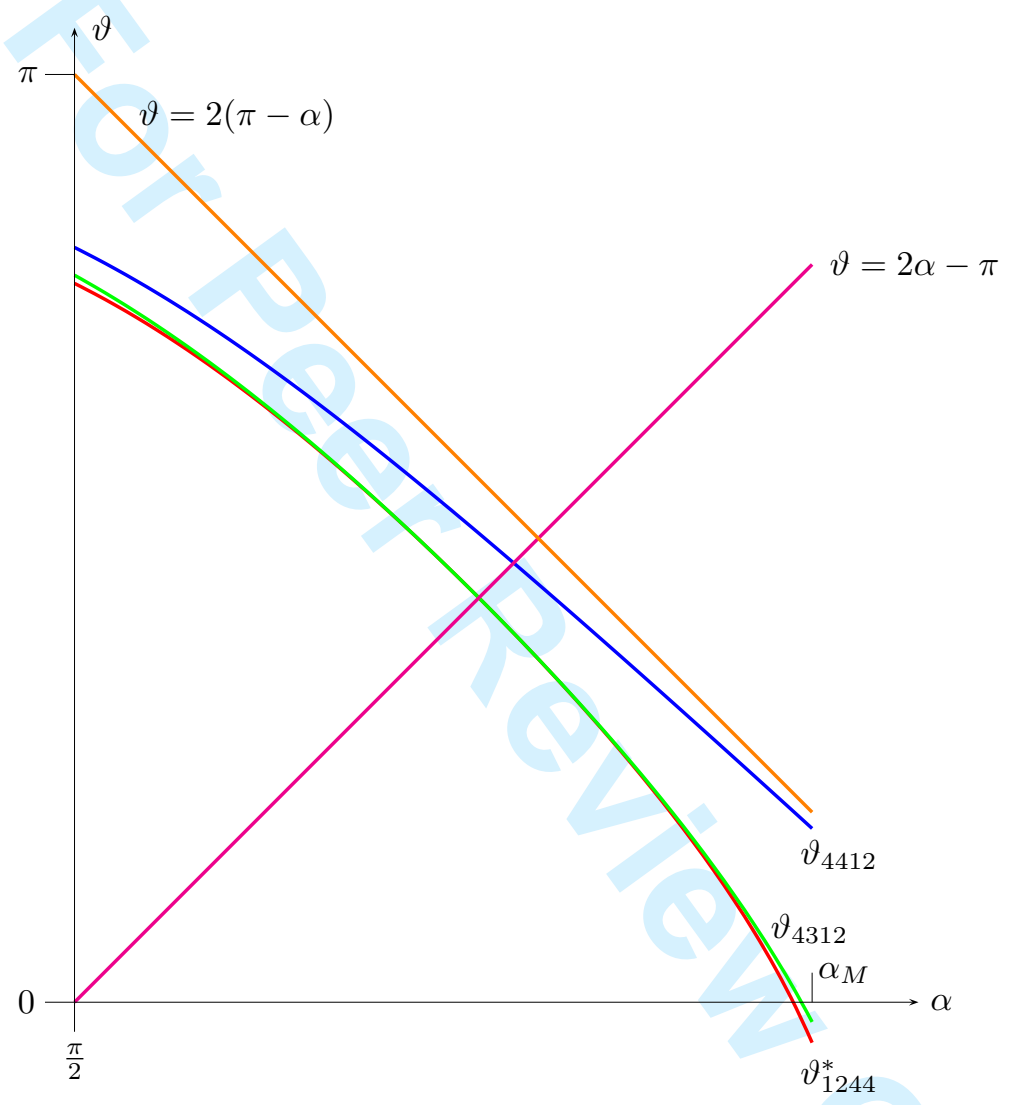



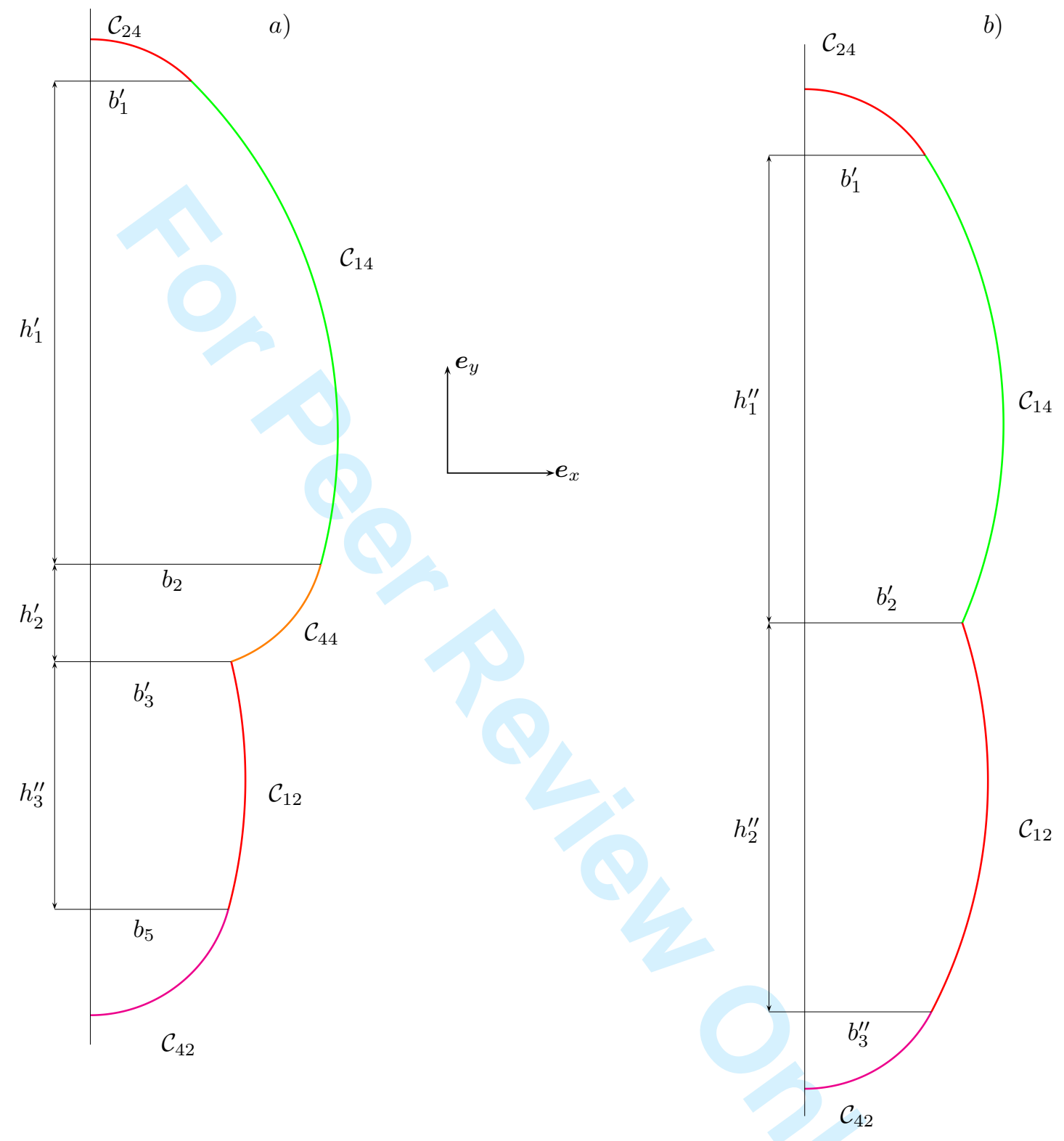


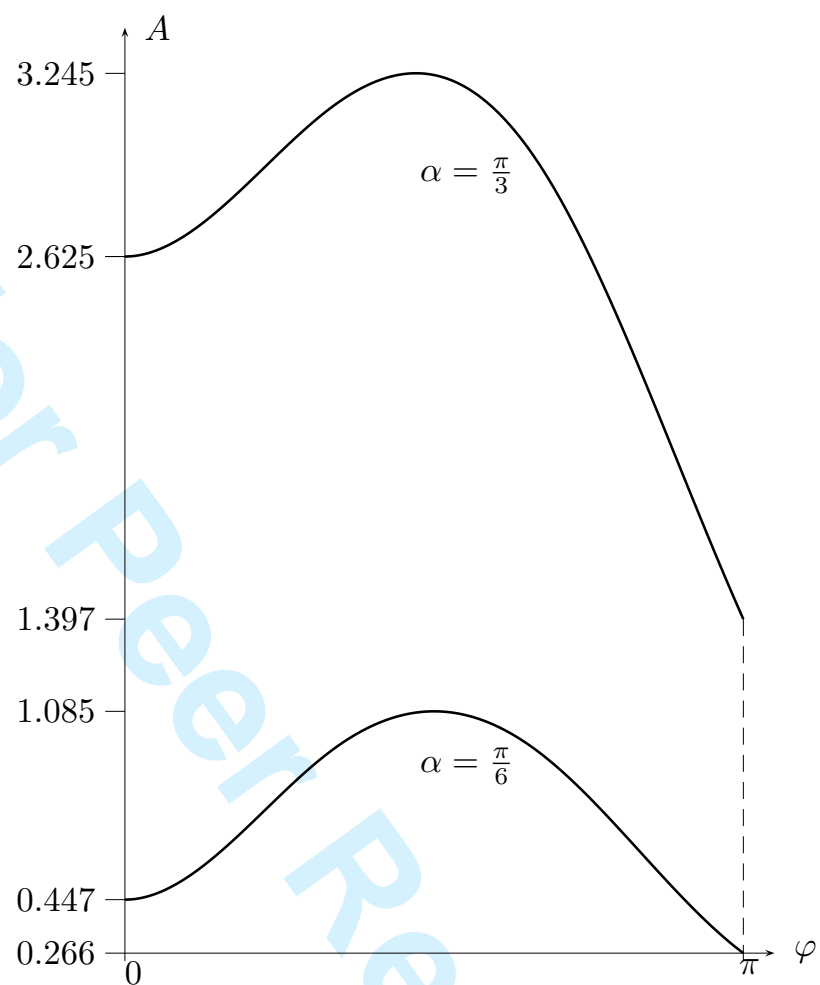


Page 39 of 42Molecular-Rhysics

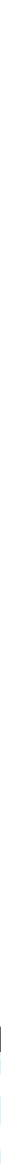




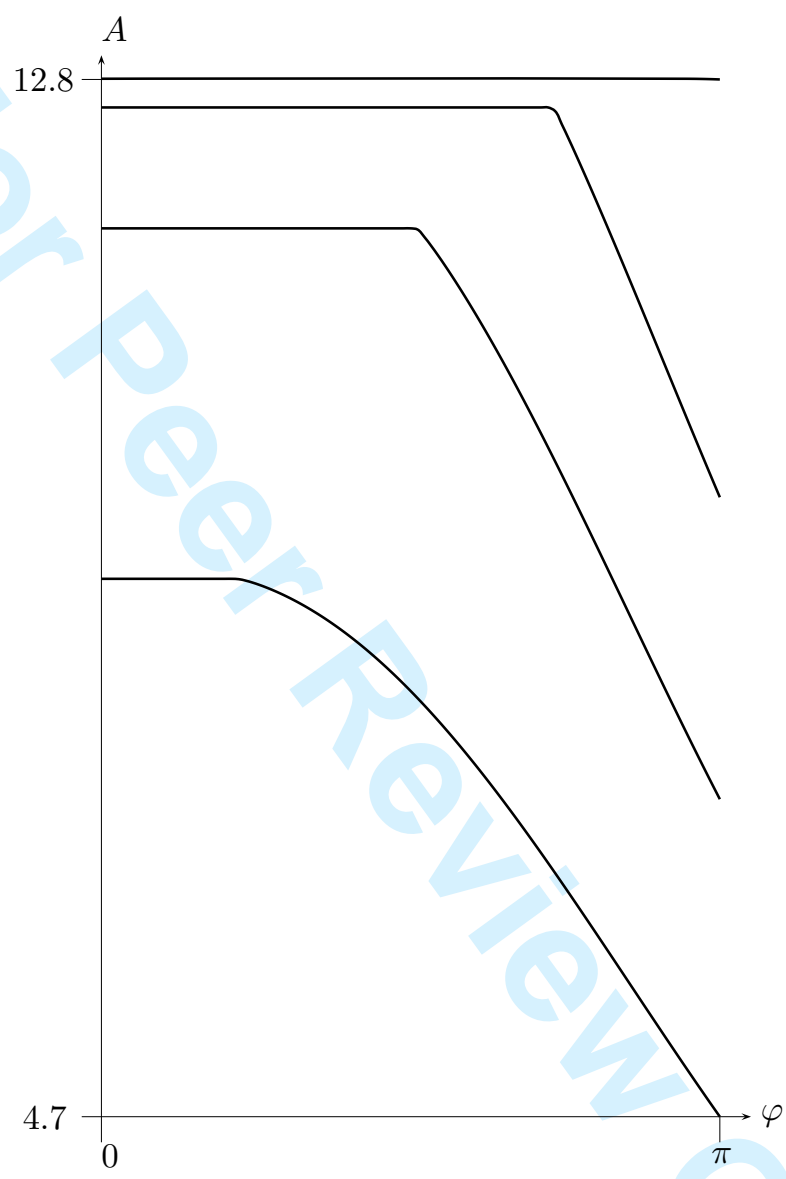




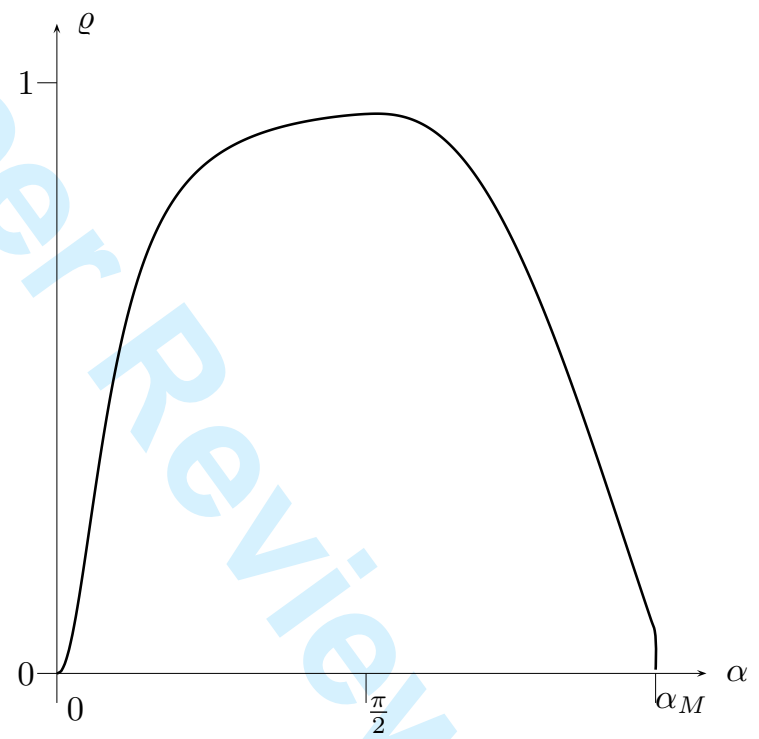

40 41 42 43 


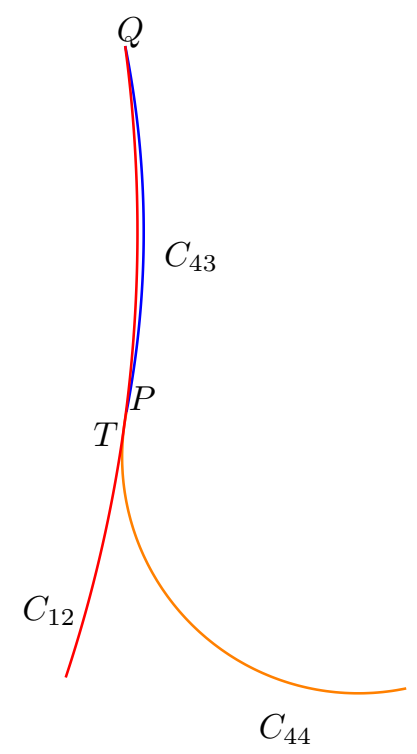

